\title{
Modulation of Camassa-Holm equation and reciprocal transformations
}

\author{
Simonetta Abenda \\ Dipartimento di M atematica e CIRAM \\ Università degli Studi di Bologna, Italy \\ abenda@ciram.unibo.it \\ and \\ Tamara Grava \\ SISSA, Via Beirut 9, Trieste, Italy \\ grava@sissa.it
}

October 1, 2018

Dedicated to Pierre van Moerbeke on his sixtieth birthday

\begin{abstract}
We derive the modulation equations or Whitham equations for the CamassaHolm $(\mathrm{CH})$ equation. We show that the modulation equations are hyperbolic and admit bi-Hamiltonian structure. Furthermore they are connected by a reciprocal transformation to the modulation equations of the first negative flow of the Korteweg de Vries ( $\mathrm{KdV}$ ) equation. The reciprocal transformation is generated by the Casimir of the second Poisson bracket of the KdV averaged flow. We show that the geometry of the bi-Hamiltonian structure of the KdV and $\mathrm{CH}$ modulation equations is quite different: indeed the $\mathrm{KdV}$ averaged bi-Hamiltonian structure can always be related to a semisimple Frobenius manifold while the $\mathrm{CH}$ one cannot.
\end{abstract}




\section{Introduction}

In 1993 R. Camassa and D. Holm [7] proposed a new equation

$$
\mathrm{u}_{t}+3 \mathrm{uu}_{x}=\left(\mathrm{u}_{x x t}+2 \mathrm{u}_{x} \mathrm{u}_{x x}+\mathrm{uu}_{x x x}\right)-2 \nu \mathrm{u}_{x},
$$

with $\nu$ a constant parameter, deriving it as the governing equation for waves in shallow water when surface tension is present. This involves an asymptotic expansion in small amplitude of the incompressible Euler equation for unidirectional motion under the influence of gravity that extends one order beyond the Korteweg-de Vries $(\mathrm{KdV})$ equation. (1I) is also an element in a class of equations introduced by A.Fokas and B.Fuchssteiner [20] through the method of recursion operators in 1981.

Equation (10) is strongly nonlinear, admits a bi-Hamiltonian structure [20], a Lax pair [7] and it is formally integrable through the inverse scattering method [9]. The bi-Hamiltonian structure of the $\mathrm{CH}$ equation can be described as follows

$$
m_{t}=P_{1} \frac{\delta H_{2}}{\delta m}, \quad m=\mathrm{u}-\mathrm{u}_{x x}, \quad P_{1}=-\partial_{x}+\partial_{x}^{3},
$$

with

$$
H_{2}=\frac{1}{2} \int\left(\mathrm{u}^{3}+\mathrm{uu}_{x}^{2}+2 \nu \mathrm{u}^{2}\right) d x,
$$

or

$$
m_{t}=P_{2} \frac{\delta H_{1}}{\delta m}, \quad P_{2}=-\partial_{x} m-m \partial_{x}-2 \nu \partial_{x},
$$

and

$$
H_{1}=\frac{1}{2} \int\left(\mathrm{u}^{2}+\mathrm{u}_{x}^{2}\right) d x .
$$

The bi-Hamiltonian structure implies that the $\mathrm{CH}$ equation has an infinite number of conserved quantities. The functionals $H_{k}, k \in \mathbb{Z}$, defined by

$$
P_{2} \frac{\delta H_{k}}{\delta m}=P_{1} \frac{\delta H_{k+1}}{\delta m}, \quad k \in \mathbb{N},
$$

are conserved quantities in involution with respect to the Poisson bracket determined either by $P_{1}$ or $P_{2}$. The Hamiltonian $H_{0}=\int m d x$ is the Casimir of the first Poisson tensor $P_{1}$. For $k>2$ the Hamiltonian densities of $H_{k}$ are not local functions of $\mathrm{u}$ and its spatial derivatives.

In the case $\nu=0, \mathrm{R}$. Camassa and D. Holm [7] proved the existence of solutions that are continuous but only piece-wise analytic (peakons). The $\mathrm{CH}$ equation 
possesses soliton solutions, periodic finite-gap solutions [8, 10, 4, real finite-gap solutions 22 and, for $\nu=0$, multi-peakons 6]. In particular, the algebro-geometric solutions of $(\mathrm{CH})$ are described as Hamiltonian flows on nonlinear subvarieties (strata) of generalized Jacobians. This implies that the associated finite dimensional integrable systems may be described in the framework of integrable systems with deficiency [36, 1] whose algebraic-geometrical structure has much in common with the celebrated algebraically completely integrable systems introduced and thoroughly studied by M. Adler and P. van Moerbeke 3 .

In this work we derive the Whitham modulation equations for the $\mathrm{CH}$ flows. Whitham modulation equations for a nonlinear evolution system describe slow modulations of parameters over a family of periodic travelling wave solutions (or families of multi-phase solutions which are so far known to exist only for integrable systems). Contrary to the Korteweg de Vries case [26, it is an open problem to show that the Cauchy problem for $\mathrm{CH}$ with slowly varying initial data is described by the Whitham equations. Both for $\mathrm{KdV}$ and $\mathrm{CH}$ equations, this approximation is physically meaningful when the ratio between the water depth and the wavelength is very small [15].

The Whitham equations are a system of hydrodynamic type equations [12] and in the Riemann invariant coordinates take the form

$$
u_{t}^{i}+v^{i}(\boldsymbol{u}) u_{x}^{i}=0, \quad i=1, \ldots, N, \quad \boldsymbol{u}=\left(u^{1}, \ldots, u^{N}\right),
$$

where we denote fast and slow variables with the same letters $x$ and $t$ and upper indeces denote controvariant vectors. The original evolution system is usually Lagrangian or Hamiltonian and this property is usually inherited by the equations of slow modulations. To average the original equations in the Lagrangian form, Whitham [37] introduced the pseudo-phase method and then he constructed the corresponding Hamiltonian structure. For local Hamiltonian structures, B.A. Dubrovin and S.P. Novikov 12 introduced a procedure for averaging local Poisson brackets and obtained the corresponding modulation equations. A third method to derive the Whitham modulation equations is the nonlinear analog of the WKB method [11. It can be proven that the three methods lead to the same equations for the case in which the original equation has a local Hamiltonian structure and local Hamiltonian densities (see [12] and references therein).

The Camassa-Holm equation does not possess a local Hamiltonian structure: indeed in the variable $\mathrm{u}$ the Hamiltonian operator is strongly nonlocal and the Hamiltonian densities of $H_{k}$ are non-local for $k>2$. In the variable $m$ the Hamiltonian densities of $H_{k}$ are nonlocal for $k>0$. Therefore the $\mathrm{CH}$ equation does not 
fit into the method of averaging local Hamiltonian structure [12] nor even in the Maltsev-Novikov method of averaging weakly nonlocal Hamiltonian structures [28] (an Hamiltonian structure is weakly nonlocal if it is polynomial in $\partial_{x}$ and its higher derivatives and linear in $\partial_{x}^{-1}$ ) or in the Maltsev method [29] of averaging weakly non-local symplectic form (inverse of the Hamiltonian operator). The latter method applies to Camassa-Holm only when averaging one-phase solutions 30.

The $\mathrm{CH}$ equation can be written as a local Lagrangian system and we use the Whitham method (modulation equations in Lagrangian form) to derive the modulation equations for the one-phase periodic solution. The $\mathrm{CH}$ modulation equations for the Riemann invariants $u^{1}<u^{2}<u^{3}$, take the form

$$
\partial_{t} u^{i}+C^{i}(\boldsymbol{u}) \partial_{x} u^{i}=0, \quad i=1, \ldots, 3,
$$

where

$$
\begin{aligned}
& C^{1}\left(u^{1}, u^{2}, u^{3}\right)=u^{1}+u^{2}+u^{3}+2 \nu+2 \frac{\left(u^{1}+\nu\right)\left(u^{1}-u^{2}\right) \Lambda(K(s), \rho, s)}{\left(u^{2}+\nu\right)[K(s)-E(s)]} \\
& C^{2}\left(u^{1}, u^{2}, u^{3}\right)=u^{1}+u^{2}+u^{3}+2 \nu+\frac{2\left(u^{2}-u^{1}\right) \Lambda(K(s), \rho, s)}{K(s)-\frac{\left(u^{2}+\nu\right)\left(u^{3}-u^{1}\right)}{\left(u^{1}+\nu\right)\left(u^{3}-u^{2}\right)} E(s)} \\
& C^{3}\left(u^{1}, u^{2}, u^{3}\right)=u^{1}+u^{2}+u^{3}+2 \nu+2 \frac{\left(u^{1}+\nu\right)\left(u^{3}-u^{2}\right) \Lambda(K(s), \rho, s)}{\left(u^{2}+\nu\right) E(s)} .
\end{aligned}
$$

In the above formulas $K(s)$ and $E(s)$ are the complete elliptic integrals of the first and second kind with modulus $s^{2}=\frac{\left(u^{2}-u^{1}\right)\left(u^{3}+\nu\right)}{\left(u^{3}-u^{1}\right)\left(u^{2}+\nu\right)}$ and $\Lambda(K(s), \rho, s)$ is the complete elliptic integral of the third kind defined by

$$
\Lambda(K(s), \rho, s)=\int_{0}^{K(s)} \frac{d v}{1-\rho^{2} s n^{2} v}, \quad \rho^{2}=\frac{u^{2}-u^{1}}{u^{2}+\nu},
$$

with $s n$ the Jacobi elliptic function. The equations are hyperbolic for $-\nu<u^{1}<$ $u^{2}<u^{3}$ where $\nu$ is the parameter entering in the $\mathrm{CH}$ equation (11).

Then following Hayes [25] and Whitham [37] the equations can be written in Hamiltonian form with a local Poisson bracket of Dubrovin-Novikov type

$$
u_{t}^{i}=-C^{i}(\boldsymbol{u}) u_{x}^{i}=A^{i j} \frac{\partial h}{\partial u^{j}}
$$

where

$$
A^{i j}=g^{i i} \delta^{i j} \frac{d}{d x}-g^{i i} \Gamma_{i k}^{j} u_{x}^{k}
$$


is the Hamiltonian operator and $h$ the Hamiltonian density. As pointed out by Dubrovin and Novikov, $A^{i j}$ defines a Hamiltonian operator if and only if $g^{i i}=g^{i i}(\boldsymbol{u})$ is a flat non degenerate metric and $\Gamma_{i k}^{j}$ are the Christoffel symbols of the corresponding Levi-Civita connection. We also find a second local compatible Hamiltonian structure which is obtained from the flat metric $g^{i i}(\boldsymbol{u})\left(u^{i}+\nu\right)$ where $\nu$ is the constant in the $\mathrm{CH}$ equation. Therefore the nonlocal bi-Hamiltonian structure of the original $\mathrm{CH}$ equation averages to a local bi-Hamiltonian structure of Dubrovin Novikov type.

A reciprocal transformation is a closed form which changes the independent variables of the equation and maps conservation laws into conservations laws, but it does not preserve the Poisson structure as shown by E.V. Ferapontov and M.V. Pavlov [18, [17]. The Camassa Holm equation can be transformed by a reciprocal transformation into the first negative flow of the KdV hierarchy 21 (also known as AKNS equation [2]). An elegant treatment of the relations among positive and negative flows of the $\mathrm{CH}$ and $\mathrm{KdV}$ hierarchies can be found in [27].

Let $g_{i i}^{K d V}$ and $g_{i i}^{K d V} / \beta^{i}$ be the flat compatible metrics associated to the biHamiltonian structure of the KdV modulation equations [35] 13] with respect to the usual Riemann invariants $\beta^{1}, \beta^{2}, \beta^{3}$ as defined in [37. Then the reciprocal transformation is generated by the Casimir $\mathcal{H}_{0}$ of the Hamiltonian operator associated to the metric $g_{i i}^{K d V} / \beta^{i}$. According to the results in [18, [17] the reciprocal transformation maps the two $\mathrm{KdV}$ flat metrics to the $\mathrm{CH}$ metrics

$$
\frac{g_{i i}^{K d V}}{\mathcal{H}_{0}^{2}}, \quad \frac{g_{i i}^{K d V}}{\mathcal{H}_{0}^{2} \beta^{i}}
$$

which are not flat. The relation between the CH Riemann invariants and the KdV Riemann invariants is $\beta^{i}=1 /\left(u^{i}+\nu\right)$. The corresponding $\mathrm{CH}$ modulation equations are Hamiltonian with respect to two non-local operators of Mokhov-Ferapontov and Ferapontov type [16, 17] which are of the form (5) plus a nonlocal tail. However from the Lagrangian averaging, we independently prove the existence of one local Hamiltonian structure. We show that the two metrics

$$
\frac{g_{i i}^{K d V}}{\mathcal{H}_{0}^{2}\left(\beta^{i}\right)^{2}}, \quad \frac{g_{i i}^{K d V}}{\mathcal{H}_{0}^{2}\left(\beta^{i}\right)^{3}}
$$

are flat and define a flat pencil, that is, the $\mathrm{CH}$ modulation equations are biHamiltonian with respect to two local Hamiltonian operators of the form (5). We remark that the two flat $\mathrm{KdV}$ metrics $g_{i i}^{K d V}$ and $g_{i i}^{K d V} / \beta^{i}$ are related to a semisimple Frobenius manifold [14. More in general, B. Dubrovin 14] proves that, under 
certain assumptions, a flat pencil of contravariant metrics on a manifold induces a Frobenius structure on it. One of the assumptions is the requirement that one of the two flat metrics is of Egorov type (namely its rotation coefficients are symmetric). Since none of the two $\mathrm{CH}$ flat metrics have the Egorov property, there is no Frobenius structure associated to this system. Therefore, from the geometric point of view, the $\mathrm{KdV}$ modulation equations and the $\mathrm{CH}$ modulation equation belong to two different classes.

All the results presented here for the one-phase $\mathrm{CH}$ modulation equations may be generalized to the multi-phase case in a straightforward way. However, in the present paper we have decided to concentrate only on the one-phase case to better clarify

similarities and differences with the $\mathrm{KdV}$ case, and we will present the discussion of the multi-phase case in a future publication.

\section{Whitham modulation equations}

In this section we use Lagrangian formalism to average the Camassa-Holm equation in the genus one case and refer to [12, 31] for a general exposition of the method we use. Introducing the potential

$$
\phi: \phi_{x}=\mathrm{u},
$$

equation (11) takes the form

$$
\phi_{x t}-\phi_{x x x t}+3 \phi_{x} \phi_{x x}-2 \phi_{x x} \phi_{x x x}-\phi_{x} \phi_{x x x x}+2 \nu \phi_{x x}=0
$$

and a Lagrangian is

$$
\mathcal{L}=-\frac{1}{2} \phi_{x} \phi_{t}+\frac{1}{2} \phi_{x x x} \phi_{t}-\frac{1}{2} \phi_{x}^{3}-\nu \phi_{x}^{2}+\frac{1}{4} \phi_{x}^{2} \phi_{x x x}
$$

We consider $2 \pi$-periodic solutions of the form

$$
\mathrm{u}=\eta(\theta), \quad \theta=k x-\omega t
$$

Following Whitham 37, we introduce the pseudo-phase

$$
\phi=\psi+\Phi(\theta), \quad \psi=\beta x-\gamma t, \quad \theta=k x-\omega t,
$$


where $\Phi(\theta)$ is a $2 \pi$ periodic function of $\theta$ with zero average. The averaged Lagrangian over the one-dimensional real torus is

$$
\begin{aligned}
\overline{\mathcal{L}} & =\oint d \theta\left[-\frac{1}{2}\left(\beta+k \Phi_{\theta}\right)\left(\gamma-\omega \Phi_{\theta}\right)+\frac{1}{2} k^{3} \Phi_{\theta \theta \theta}\left(\gamma-\omega \Phi_{\theta}\right)-\right. \\
& \left.-\frac{1}{2}\left(\beta+k \Phi_{\theta}\right)^{3}-\nu\left(\beta+k \Phi_{\theta}\right)^{2}+\frac{1}{4}\left(\beta+k \Phi_{\theta}\right)^{2} k^{3} \Phi_{\theta \theta \theta}\right]
\end{aligned}
$$

Now we suppose that the constants $\beta, k, \gamma$ and $\omega$ are slowly varying functions of time, that is $\beta=\beta(X, T), \gamma=\gamma(X, T), k=k(X, T)$ and $\omega=\omega(X, T)$ with $X$ and $T$ "slow variables". Then

$$
\overline{\mathcal{L}}=\overline{\mathcal{L}}(k, \omega, \beta, \gamma ; X, T) .
$$

The equations of slow modulation of the parameters $k, \omega, \beta, \gamma$ are the extremals of the functional 37.

$$
\int \overline{\mathcal{L}}(k, \omega, \beta, \gamma) d X d T
$$

and take the form

$$
\begin{cases}\partial_{X} \overline{\mathcal{L}}_{k}+\partial_{T} \overline{\mathcal{L}}_{\omega}=0, & k_{T}+\omega_{X}=0 \\ \partial_{X} \overline{\mathcal{L}}_{\gamma}+\partial_{T} \overline{\mathcal{L}}_{\beta}=0, & \beta_{T}+\gamma_{X}=0\end{cases}
$$

As in the KdV case, substituting the forth equation into the third in (17), the $X$ derivative disappears and we get a constraint. Thus the number of equations reduces from four to three.

We get to the same conclusion transforming the modulation equations in Hamiltonian form. Following Hayes [25] and Whitham [37, let us introduce the Hamiltonian density

$$
\mathcal{H}=\mathcal{H}(k, \omega, \beta, \gamma)=\omega \overline{\mathcal{L}}_{\omega}+\gamma \overline{\mathcal{L}}_{\gamma}-\overline{\mathcal{L}}
$$

Then the modulation equations are Hamiltonian with respect to the canonical Poisson bracket

$$
\left\{k(X), \overline{\mathcal{L}}_{\omega}(Y)\right\}=\delta^{\prime}(X-Y), \quad\left\{\beta(X), \overline{\mathcal{L}}_{\gamma}(Y)\right\}=\delta^{\prime}(X-Y) .
$$

Since the constraint

$$
\overline{\mathcal{L}}_{\gamma}=\frac{1}{2} \beta
$$

the number of fields reduces from four to three. This is connected with the Dirac reduction. Therefore the Whitham equations can be written in Hamiltonian form with a local Dubrovin-Novikov Poisson bracket [30]

$$
\left\{k(X), \overline{\mathcal{L}}_{\omega}(Y)\right\}=\delta^{\prime}(X-Y), \quad\{\beta(X), \beta(Y)\}=2 \delta^{\prime}(X-Y)
$$


and with Hamiltonian $\mathcal{H}=\mathcal{H}(k, J, \beta), J=\overline{\mathcal{L}}_{\omega}$. In these variables the equations of motion are Hamiltonian

$$
k_{T}=\partial_{X} \mathcal{H}_{J}, \quad J_{T}=\partial_{X} \mathcal{H}_{k}, \quad \beta_{T}=\partial_{X} \mathcal{H}_{\beta}
$$

\section{Modulation equations for $\mathrm{CH}$ in Riemann in- variant form}

The modulation equations (8) can also be written in Riemann invariant form. For the purpose we introduce the spectral curve associated to the periodic travelling wave solution $\mathrm{u}(x, t)=\eta(k x-\omega t)$. When we plug $\eta(\theta), \theta=k x-\omega t$, into the $\mathrm{CH}$ equation (11), we get, after integration,

$$
k^{2}(c-\eta) \eta_{\theta}^{2}+(2 \nu-c) \eta^{2}+\eta^{3}+2 B \eta-2 A=0,
$$

where $A$ and $B$ are constants of integration and $c=\omega / k$. The $\mathrm{CH}$ one-phase solution $\mathrm{u}(x, t)=\eta(k x-\omega t)$ is obtained by inverting the third kind differential

$$
\int_{u_{0}}^{u} \frac{(\eta-c) d \eta}{\sqrt{(\eta-c)\left(\eta^{3}+(2 \nu-c) \eta^{2}+2 B \eta-2 A\right)}}=k x-\omega t .
$$

The inversion of the above integral is discussed in [4] where the solution $\mathrm{u}(x, t)$ is expressed in terms of convenient generalized theta-functions in two variables, which are constrained to the generalized theta-divisor. Integration of (9) over $\theta$ yields the amplitude dependent dispersion relation for the nonlinear dispersive wave

$$
k \oint \frac{(\eta-c) d \eta}{\sqrt{(\eta-c)\left(\eta^{3}+(2 \nu-c) \eta^{2}+2 B \eta-2 A\right)}}=2 \pi,
$$

where the integration is taken on a closed path between $e^{2}$ and $e^{1}$ where $c>e^{1}>$ $e^{2}>e^{3}$ are the roots of the polynomial

$$
\eta^{3}-(c-2 \nu) \eta^{2}+2 B \eta-2 A
$$

with the constraint

$$
2 \nu=c-e^{1}-e^{2}-e^{3} .
$$

Here and below, we denote vectors with upper indices.

¿From now on, we will use small letters $x, t$ for the 'slow variables' $X, T$ introduced in the previous section, since we deal only with modulation equations and no ambiguity may occur. 
Theorem 3.1 The one-phase CH modulation equations (8) take the Riemann invariant form

$$
\partial_{t} u^{i}+C^{i}(\boldsymbol{u}) \partial_{x} u^{i}=0, \quad i=1, \ldots, 3,
$$

where the Riemann invariants $\boldsymbol{u}=\left(u^{1}, u^{2}, u^{3}\right), u^{1}<u^{2}<u^{3}$, are

$$
u^{1}=\frac{1}{2}\left(e^{2}+e^{3}\right), \quad u^{2}=\frac{1}{2}\left(e^{1}+e^{3}\right), \quad u^{3}=\frac{1}{2}\left(e^{1}+e^{2}\right),
$$

and the speeds $C^{i}(\boldsymbol{u})$ take the form

$$
C^{i}(\boldsymbol{u})=\frac{\partial_{u^{i}} \omega(\boldsymbol{u})}{\partial_{u^{i}} k(\boldsymbol{u})} .
$$

The wave number and frequency are given by the Abelian integrals

$$
\begin{aligned}
& k=2 \pi\left(\oint_{a} \frac{(\lambda+\nu) d \lambda}{\sqrt{R(\lambda)}}\right)^{-1}, R(\lambda)=(\lambda+\nu)\left(\lambda-u^{1}\right)\left(\lambda-u^{2}\right)\left(\lambda-u^{3}\right), \\
& \omega=\left(2 \nu+u^{1}+u^{2}+u^{3}\right) k
\end{aligned}
$$

and the integration is taken on cycle a passing between $u^{2}$ and $u^{1}$ (see figure 11).

The existence of the Riemann invariants and equations (12) is proven directly starting from (8) and using variational identities of Abelian integrals. To write the velocities in an explicit form, we introduce the integrals

$$
I_{k}=\oint_{a} \frac{\lambda^{k}}{\sqrt{R(\lambda)}} d \lambda, \quad k \geq 0,
$$

where $R(\lambda)$ is defined in (13).

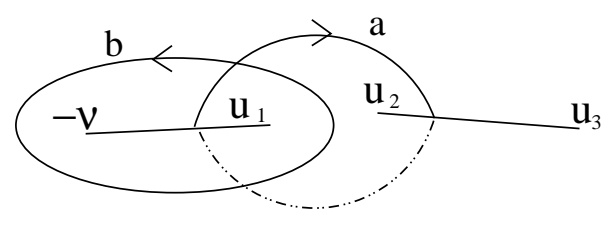

Figure 1: The homology basis.

Let $\sigma_{1}(\lambda)$ be the normalized third kind differential with first order pole at $(\infty, \pm \infty)$ with residue \pm 1 , respectively, and let $\sigma_{2}$ be the normalized second kind 
differential with second order pole at infinity, namely

$$
\begin{array}{ll}
\sigma_{1}(\lambda)=\frac{P_{1}(\lambda) d \lambda}{\sqrt{R(\lambda)}}, & P_{1}(\lambda)=\lambda+\gamma_{1} \\
\sigma_{2}(\lambda)=\frac{P_{2}(\lambda) d \lambda}{\sqrt{R(\lambda)}}, & P_{2}(\lambda)=\lambda^{2}-\frac{1}{2}\left(u^{1}+u^{2}+u^{3}-\nu\right) \lambda+\gamma_{2}
\end{array}
$$

where the constants $\gamma_{1}=-\frac{I_{1}}{I_{0}}$ and $\gamma_{2}=-\frac{I_{2}}{I_{0}}+\frac{1}{2}\left(u^{1}+u^{2}+u^{3}-\nu\right) \frac{I_{1}}{I_{0}}$ are uniquely determined by

$$
\oint_{a} \sigma_{i}(\lambda)=0, \quad i=1,2
$$

These constants are explicitly given by

$$
\gamma_{2}=\frac{1}{2}\left[u^{1} u^{2}-\nu u^{3}+\left(u^{3}-u^{1}\right)\left(u^{2}+\nu\right) \frac{E(s)}{K(s)}\right], \quad \gamma_{1}=\nu-\left(u^{1}+\nu\right) \frac{\Lambda(K(s), \rho, s)}{K(s)},
$$

where

$$
K(s)=\int_{0}^{\pi / 2} \frac{d \psi}{\sqrt{1-s^{2} \sin ^{2} \psi}}, \quad E(s)=\int_{0}^{\pi / 2} d \psi \sqrt{1-s^{2} \sin ^{2} \psi}
$$

are the complete elliptic integrals of the first and second kind respectively with modulus

$$
s^{2}=\frac{\left(u^{2}-u^{1}\right)\left(u^{3}+\nu\right)}{\left(u^{3}-u^{1}\right)\left(u^{2}+\nu\right)}
$$

and

$$
\Lambda(K(s), \rho, s)=\int_{0}^{K(s)} \frac{d v}{1-\rho^{2} s n^{2} v}, \quad \rho^{2}=\frac{u^{2}-u^{1}}{u^{2}+\nu}
$$

is the complete elliptic integral of the third kind with $s n$ the Jacobi elliptic function.

Theorem 3.2 The speeds $C^{i}(\boldsymbol{u}), i=1,2,3$ defined in (12) take the form

$$
\begin{aligned}
& C^{1}\left(u^{1}, u^{2}, u^{3}\right)=u^{1}+u^{2}+u^{3}+2 \nu+2 \frac{\left(u^{1}+\nu\right)\left(u^{1}-u^{2}\right) \Lambda(K(s), \rho, s)}{\left(u^{2}+\nu\right)[K(s)-E(s)]} \\
& C^{2}\left(u^{1}, u^{2}, u^{3}\right)=u^{1}+u^{2}+u^{3}+2 \nu+\frac{2\left(u^{2}-u^{1}\right) \Lambda(K(s), \rho, s)}{K(s)-\frac{\left(u^{2}+\nu\right)\left(u^{3}-u^{1}\right)}{\left(u^{1}+\nu\right)\left(u^{3}-u^{2}\right)} E(s)} \\
& C^{3}\left(u^{1}, u^{2}, u^{3}\right)=u^{1}+u^{2}+u^{3}+2 \nu+2 \frac{\left(u^{1}+\nu\right)\left(u^{3}-u^{2}\right) \Lambda(K(s), \rho, s)}{\left(u^{2}+\nu\right) E(s)} .
\end{aligned}
$$


where $K(s), E(s)$ and $\Lambda(K(s), \rho, s)$ are the complete elliptic integrals of first, second and third kind with modulus $s^{2}=\frac{\left(u^{2}-u^{1}\right)\left(u^{3}+\nu\right)}{\left(u^{3}-u^{1}\right)\left(u^{2}+\nu\right)}$.

The equations $\partial_{t} u^{i}+C^{i}(\boldsymbol{u}) \partial_{x} u^{i}=0$ are hyperbolic and the velocities satisfy

$$
C^{1}(\boldsymbol{u})<C^{3}(\boldsymbol{u}), \quad C^{2}(\boldsymbol{u})<C^{3}(\boldsymbol{u}), \quad-\nu<u^{1}<u^{2}<u^{3} .
$$

In the limit when two Riemann invariants coalesce, the modulation equation reduce to the dispersionless $\mathrm{CH}$ equation

$$
\partial_{t} \mathrm{u}+(3 \mathrm{u}+2 \nu) \partial_{x} \mathrm{u}=0
$$

To prove the theorem we introduce the normalized holomorphic differential $\phi(\lambda)$

$$
\phi(\lambda)=\frac{d \lambda}{I_{0} \sqrt{R(\lambda)}}, \quad \oint_{a} \phi(\lambda)=1 .
$$

Next we observe that the wave number $k$ defined in (13) takes the form

$$
k=-2 \pi \frac{\phi(-\nu)}{\sigma_{1}(-\nu)}
$$

where

$$
\sigma_{1}(-\nu):=\frac{2 P_{1}(-\nu)}{\sqrt{\left(-\nu-u^{1}\right)\left(-\nu-u^{2}\right)\left(-\nu-u^{3}\right)}}=\left.\frac{\sigma_{1}(\lambda)}{d t}\right|_{\lambda=-\nu}, \quad t^{2}=\lambda+\nu
$$

and

$$
\phi(-\nu):=\frac{2}{I_{0} \sqrt{\left(-\nu-u^{1}\right)\left(-\nu-u^{2}\right)\left(-\nu-u^{3}\right)}}=\left.\frac{\phi(\lambda)}{d t}\right|_{\lambda=-\nu}, \quad t^{2}=\lambda+\nu
$$

with $\sigma_{1}(\lambda)$ defined in (15). The following variational formulas hold [23]

$$
\frac{\partial}{\partial u^{i}} \phi(-\nu)=\frac{1}{2} \phi\left(u^{i}\right) \Omega_{\nu}\left(u^{i}\right), \quad \frac{\partial}{\partial u^{i}} \sigma_{1}(-\nu)=\frac{1}{2} \sigma_{1}\left(u^{i}\right) \Omega_{\nu}\left(u^{i}\right),
$$

where

$$
\phi\left(u^{i}\right):=\left.\frac{\phi(\lambda)}{d t}\right|_{\lambda=u^{i}}, \Omega_{\nu}\left(u^{i}\right):=\left.\frac{\Omega_{\nu}(\lambda)}{d t}\right|_{\lambda=u^{i}}, \quad t^{2}=\lambda-u^{i}
$$


and $\Omega_{\nu}(\lambda)$ is a second kind Abelian differential with second order pole at $\lambda=-\nu$ with asymptotic behavior for $\lambda \rightarrow-\nu$

$$
\Omega_{\nu}(\lambda) \rightarrow \frac{d t}{t^{2}}, \quad t^{2}=\lambda+\nu
$$

and normalized by the condition

$$
\oint_{a} \Omega_{\nu}(\lambda)=0
$$

The differential $\Omega_{\nu}(\lambda)$ is explicitly given by the expression

$$
\begin{aligned}
\Omega_{\nu}(\lambda) & =\frac{P_{\nu}(\lambda) d \lambda}{\sqrt{R(\lambda)} \sqrt{\left(-\nu-u^{1}\right)\left(-\nu-u^{2}\right)\left(-\nu-u^{3}\right)}}, \\
P_{\nu}(\lambda) & =\frac{-\left(\nu+u^{1}\right)\left(\nu+u^{2}\right)\left(\nu+u^{3}\right)}{2(\lambda+\nu)}+P_{2}(-\nu),
\end{aligned}
$$

where $P_{2}(\lambda)$ has been defined in (16). Inserting (20) and (21) into (12), we finally obtain the expression

$$
C^{i}(\boldsymbol{u})=u^{1}+u^{2}+u^{3}+2 \nu-\frac{P_{1}(-\nu)}{P_{\nu}\left(u^{i}\right)} \prod_{j \neq i, j=1}^{3}\left(u^{i}-u^{j}\right), i=1,2,3
$$

where the rational function $P_{1}(\lambda)$ and $P_{\nu}(\lambda)$ are as in (15) and (24) respectively. Finally inserting (18) into (25) we arrive at the formula (19).

Next we prove the ordering of the velocities using the formula (25). Since $-\nu<$ $u^{1}<u^{2}<u^{3}$, and $\lim _{\lambda \rightarrow-\nu^{+}} P_{\nu}(\lambda)=-\infty$, by monotonicity, there is only one point $\lambda^{*}>-\nu$ for which

$$
\frac{\left(\nu+u^{1}\right)\left(\nu+u^{2}\right)\left(\nu+u^{3}\right)}{2\left(\lambda^{*}+\nu\right)}=P_{2}(-\nu)
$$

moreover, because of (23), the point $\lambda^{*}$ satisfies the inequality $u^{1}<\lambda^{*}<u^{2}$. Therefore

$$
P_{\nu}\left(u^{1}\right)<0, \quad P_{\nu}\left(u^{3}\right)>P_{\nu}\left(u^{2}\right)>0 .
$$

In the same way, using (17) we conclude that $P_{1}(-\nu)<0$. Using the above inequalities, it is straightforward to verify that

$$
C^{1}(\boldsymbol{u})<C^{3}(\boldsymbol{u}), \quad, C^{2}(\boldsymbol{u})<C^{3}(\boldsymbol{u}), \quad-\nu<u^{1}<u^{2}<u^{3} .
$$


In general there is not a strict ordering between $C^{1}(\boldsymbol{u})$ and $C^{2}(\boldsymbol{u})$. For example for step-like initial data the characteristics $C^{1}(\boldsymbol{u})$ and $C^{2}(\boldsymbol{u})$ do cross for $u^{1}<u^{2}<u^{3}$.

We end the proof of the theorem studying the behavior of the speeds $C^{i}(\boldsymbol{u})$, $i=1,2,3$, when two of the Riemann invariants coalesce. In the limiting case

$$
u^{2}=v-\epsilon, \quad u^{3}=v+\epsilon, \quad \epsilon \rightarrow 0
$$

we have

$$
\lim _{\epsilon \rightarrow 0} P_{1}(\lambda)=\lambda-v, \quad \lim _{\epsilon \rightarrow 0} P_{\nu}(\lambda)=\frac{1}{2(\lambda+\nu)}(\lambda-v)\left(u^{1}+\nu\right)(v+\nu),
$$

so that

$$
\lim _{\epsilon \rightarrow 0} \frac{P_{1}(-\nu)}{P_{\nu}\left(u^{1}\right)}\left(u^{1}-u^{2}\right)\left(u^{1}-u^{3}\right)=2\left(v-u^{1}\right)
$$

and

$$
\lim _{\epsilon \rightarrow 0} \frac{P_{1}(-\nu)}{P_{\nu}\left(u^{2}\right)}\left(u^{2}-u^{1}\right)\left(u^{2}-u^{3}\right)=\lim _{\epsilon \rightarrow 0} \frac{P_{1}(-\nu)}{P_{\nu}\left(u^{3}\right)}\left(u^{3}-u^{2}\right)\left(u^{3}-u^{1}\right) .
$$

Combining the above relations, we have

$$
C^{2}\left(u^{1}, v, v\right)=C^{3}\left(u^{1}, v, v\right), \quad C^{1}\left(u^{1}, v, v\right)=3 u^{1}+2 \nu,
$$

which gives the dispersionless $\mathrm{CH}$ equation $\partial_{t} u^{1}+\left(3 u^{1}+2 \nu\right) \partial_{x} u^{1}=0$. In the same way, it can be proved that, in the limit $u^{2}=u^{1}$, the speeds $C^{1}\left(u^{1}, u^{1}, u^{3}\right)=$ $C^{2}\left(u^{1}, u^{1}, u^{3}\right)$ and $C^{3}\left(u^{1}, u^{1}, u^{3}\right)=3 u^{3}+2 \nu$.

\subsection{Hamiltonian structure and integration}

In this section, we investigate the bi-Hamiltonian structure of the one-phase Whitham equations

$$
\partial_{t} u^{i}+C^{i}(\boldsymbol{u}) \partial_{x} u^{i}=0, \quad i=1, \ldots, 3,
$$

with $C^{i}(\boldsymbol{u})$ as in (12) or (25). In section 2 we have proven that the above equations are Hamiltonian with respect to a canonical Poisson bracket. In the following we show that the $\mathrm{CH}$ modulation equations are bi-Hamiltonian with respect to local Poisson brackets of Dubrovin-Novikov type.

Proposition 3.3 The speeds $C^{i}(\boldsymbol{u}), i=1,2,3$, satisfy the following relations

$$
\frac{\partial_{u^{j}} C^{i}}{C^{j}-C^{i}}=\partial_{u^{j}} \log \sqrt{g_{i i}}
$$


where $g_{i i}=g_{i i}(\boldsymbol{u})$ is the covariant metric defined by the relation

$$
g_{i i}(\boldsymbol{u})=-4\left(u^{i}+\nu\right) \frac{\operatorname{Res}_{\lambda=u^{i}}\left\{\frac{\left(\Omega_{\nu}(\lambda)\right)^{2}}{d \lambda}\right\}}{\operatorname{Res}_{\lambda=-\nu}\left\{\frac{\left(\sigma_{1}(\lambda)\right)^{2}}{d \lambda}\right\}},
$$

with $\Omega_{\nu}(\lambda)$ the second kind differential defined in (24) and $\sigma_{1}(\lambda)$ the third kind differential defined in (15). The metric $g_{i i}(\boldsymbol{u})$ is flat. The metric $g_{i i}(\boldsymbol{u})$ is defined up to multiplication by an arbitrary function $f_{i}\left(u^{i}\right)$. The metrics

$$
\frac{g_{i i}(\boldsymbol{u})}{2\left(u^{i}+\nu\right)}, \quad \frac{g_{i i}(\boldsymbol{u})}{4\left(u^{i}+\nu\right)^{2}}, \quad \frac{g_{i i}(\boldsymbol{u})}{8\left(u^{i}+\nu\right)^{3}},
$$

are respectively flat, of constant curvature $R_{i j}^{i j}=-1$ and conformally flat with curvature tensor $R_{i j}^{i j}=-C^{i}(\boldsymbol{u})-C^{j}(\boldsymbol{u})-2 \nu$. The pencil of metrics

$$
g_{i i}(\boldsymbol{u})+\lambda \frac{g_{i i}(\boldsymbol{u})}{\left(u^{i}+\nu\right)}
$$

is flat for any real $\lambda$. None of the metrics defined in (27) and in (28) is of Egorov type.

We denote the diagonal metric in covariant form by $g_{i i}$ and its inverse by $g^{i i}$. To derive (26) it is sufficient to use the variational formulas (21) and the additional one

$$
\frac{\partial}{\partial u^{i}} \Omega_{\nu}\left(u^{j}\right)=\frac{1}{2} \Omega_{\nu}\left(u^{i}\right) \Omega_{u^{i}}\left(u^{j}\right),
$$

where $\Omega_{u^{i}}(\lambda)$ is a normalized second kind differential with second order pole at $\lambda=u^{i}$. The explicit form of $\Omega_{u^{i}}(\lambda)$ can be obtained from (24) by replacing $-\nu$ by $u^{i}$. The quantities $\Omega_{\nu}\left(u^{i}\right)$ and $\Omega_{u^{i}}\left(u^{j}\right)$ are defined as in (22).

To prove the second part of the proposition we evaluate the non-zero elements of the curvature tensor $R_{i l}^{i j}$

$$
\begin{aligned}
& R_{i l}^{i j}=-\frac{1}{\sqrt{g_{i i} g_{j j}}}\left\{\partial_{l} r_{j i}-r_{j l} r_{l i}\right\}, i \neq j \neq l, \\
& R_{i j}^{i j}=-\frac{1}{\sqrt{g_{i i} g_{j j}}}\left\{\partial_{i} r_{i j}+\partial_{j} r_{j i}+\sum_{p \neq i, j} r_{p j} r_{p i}\right\} .
\end{aligned}
$$


Here $r_{i j}$ are the rotation coefficients defined by

$$
r_{i j}=\frac{\partial_{u^{i} \sqrt{g_{j j}}}}{\sqrt{g_{i i}}}, \quad i \neq j .
$$

A metric is of Egorov type if $r_{i j}=r_{j i}, \forall i \neq j$. By direct calculation we obtain

$$
r_{i j}=\frac{1}{2}\left(\frac{u^{j}+\nu}{u^{i}+\nu}\right)^{k / 2}\left(\Omega_{u^{i}}\left(u^{j}\right)-\frac{\Omega_{\nu}\left(u^{j}\right) \sigma_{1}\left(u^{i}\right)}{\sigma_{1}(-\nu)}\right), \quad k=1,0,-1,-2 .
$$

¿From the above formula it is clear that $r_{i j} \neq r_{j i}$, for all the four metrics and therefore none of them is an Egorov metric. To evaluate (30) 311) the following additional variational formulas are needed

$$
\begin{aligned}
& \frac{\partial}{\partial u^{i}} \gamma_{1}=-\frac{1}{2}+\frac{1}{4} \sigma_{1}\left(u^{i}\right) \sigma_{2}\left(u^{i}\right), \quad i=1,2,3, \\
& \frac{\partial}{\partial u^{i}} \gamma_{2}=\frac{1}{4}\left(u^{1}+u^{2}+u^{3}-\nu\right)-\frac{1}{2} u^{i}+\frac{1}{4}\left(\sigma_{2}\left(u^{i}\right)\right)^{2}, \quad i=1,2,3,
\end{aligned}
$$

where $\sigma_{1}$ and $\sigma_{2}$ have been defined in (15) and (16) respectively and

$$
\sigma_{k}\left(u^{i}\right):=\left.\frac{\sigma_{k}(\lambda)}{d t}\right|_{\lambda=u^{i}}, \quad t^{2}=\lambda-u^{i}, \quad i=1,2,3, k=1,2 .
$$

Using the variational formulas (33) we obtain that $R_{i l}^{i j}=0$ for all the four metrics and

$$
\begin{aligned}
g_{i i}(\boldsymbol{u}) & \rightarrow R_{i j}^{i j}=0, \\
\frac{g_{i i}(\boldsymbol{u})}{2\left(u^{i}+\nu\right)} \rightarrow R_{i j}^{i j} & =0, \\
\frac{g_{i i}(\boldsymbol{u})}{4\left(u^{i}+\nu\right)^{2}} \rightarrow R_{i j}^{i j} & =-1, \\
\frac{g_{i i}(\boldsymbol{u})}{8\left(u^{i}+\nu\right)^{3}} \rightarrow R_{i j}^{i j} & =-2 \nu-C^{i}(\boldsymbol{u})-C^{j}(\boldsymbol{u}) .
\end{aligned}
$$

The flatness of the pencil of metrics (29) can be obtained from the results in [13]. An elegant proof of the flatness of the metrics, valid for any genus can be obtained in a more convenient set of coordinates $u^{i} \rightarrow \frac{1}{u^{i}+\nu}$ that makes the spectral curve odd with a branch point at infinity. We use these coordinates in the next section to compare the Whitham equations for $\mathrm{CH}$ and for $\mathrm{KdV}$. 
Remark 3.4 The non-existence of a flat Egorov metric is related to the non-existence of conservation laws of the form $a_{t}=b_{x}$ and $b_{t}=c_{x}$ [32]. Furthermore the nonexistence of a flat Egorov metric implies that the $C H$ modulation equations cannot be associated to a Frobenius manifold. We recall that, under certain assumptions, a flat pencil of contravariant metrics on a manifold induces a Frobenius structure on it [14]. One of the assumptions is the requirement that one of the two flat metrics is an Egorov metric. Therefore the geometric structure of the $\mathrm{CH}$ modulation equations is substantially different from that of the KdV modulation equations.

To any flat diagonal Riemannian controvariant metric $g^{i i}$, Dubrovin and Novikov 12 associate a local homogenous Poisson bracket of hydrodynamic type

$$
\{F, G\}=\int \frac{\delta F}{\delta u^{i}} A^{i j} \frac{\delta G}{\delta u^{j}}
$$

defined by the Hamiltonian operator

$$
A^{i j}=g^{i i} \delta^{i j} \frac{d}{d x}-g^{i i} \Gamma_{i k}^{j} u_{x}^{k}
$$

Indeed in [12, they prove that $A^{i j}$ defines a Poisson tensor if and only if $g^{i i}$ is a flat non-degenerate metric and $\Gamma_{i k}^{j}$ are the Christoffel symbols of the Levi-Civita connection compatible with the metric (the metric is not necessary diagonal in their formulation). If the metric $g^{i i}$ is not flat, the Poisson tensor needs to be modified adding a non-local tail [17, [16] of the form

$$
A^{i j}=g^{i i} \delta^{i j} \frac{d}{d x}-g^{i i} \Gamma_{i k}^{j} u_{x}^{k}+c u_{x}^{i}\left(\frac{d}{d x}\right)^{-1} u_{x}^{j}
$$

for metrics of constant curvature $c$, and of the form

$$
A^{i j}=g^{i i} \delta^{i j} \frac{d}{d x}-g^{i i} \Gamma_{i k}^{j} u_{x}^{k}+\eta^{i} u_{x}^{i}\left(\frac{d}{d x}\right)^{-1} u_{x}^{j}+u_{x}^{i}\left(\frac{d}{d x}\right)^{-1} \eta^{j} u_{x}^{j}
$$

for conformally flat metrics. In the above relation the affinors $\eta^{j}$ satisfy the equations

$$
R_{i j}^{i j}=\eta^{i}+\eta^{j}
$$

where $R_{i j}^{i j}$ is the curvature tensor.

In the following let $g_{i i}(\boldsymbol{u})$ be as in (27). Let $A_{1}^{i j}$ and $A_{2}^{i j}$ be the Hamiltonian operators of the form (134) that correspond to the flat metric $g_{i i}(\boldsymbol{u})$ and to $\frac{g_{i i}(\boldsymbol{u})}{2\left(u^{i}+\nu\right)}$, 
respectively. The linear combination $A_{1}^{i j}+\lambda A_{2}^{i j}$ is an Hamiltonian operator for any $\lambda$ because $g_{i i}(\boldsymbol{u})+\lambda g_{i i}(\boldsymbol{u}) / u^{i}$ is a flat pencil of metrics for any $\lambda$.

The Hamiltonian structure obtained in (8) coincides with Hamiltonian operator $A_{1}$. Indeed the coordinates $k=k\left(u^{1}, u^{2}, u^{3}\right), \beta=\beta\left(u^{1}, u^{2}, u^{3}\right)$ and $J=J\left(u^{1}, u^{2}, u^{3}\right)$ defined in (8) are the densities of the Casimirs for the Hamiltonian operator $A_{1}^{i j}$, namely

$$
A_{1}^{i j} \frac{\delta k}{\delta u^{j}}=A_{1}^{i j} \frac{\delta \beta}{\delta u^{j}}=A_{1}^{i j} \frac{\delta J}{\delta u^{j}}=0,
$$

and therefore they are the flat coordinates for the metric $g_{i i}(\boldsymbol{u})[12$. Defining

$$
h_{0}:=\oint m d \theta=\beta=-2 \frac{P_{2}(-\nu)}{P_{1}(-\nu)}-\nu
$$

where $P_{1}$ and $P_{2}$ have been defined in (15) and (16) respectively, we obtain the Hamiltonian densities $h_{k}=h_{k}(\boldsymbol{u}), k \geq 0$, by the recursion relation

$$
A_{2}^{i j} \frac{\partial h_{k}}{\partial u^{j}}=A_{1}^{i j} \frac{\partial h_{k+1}}{\partial u^{j}}, \quad k \geq 0 .
$$

The $\mathrm{CH}$ modulation hierarchy takes the bi-Hamiltonian form

$$
\partial_{t_{k}} u^{i}=A_{1}^{i j} \frac{\delta h_{k+1}}{\delta u^{j}}=A_{2}^{i j} \frac{\delta h_{k}}{\delta u^{j}}, \quad i=1,2,3 .
$$

Theorem 3.5 For $k=1$ and $t_{1}=t$, the above equations coincide with the modulation equations obtained in (11) and take the bi-Hamiltonian form

$$
u_{t}^{i}=A_{1}^{i j} \frac{\delta h_{2}}{\delta u^{j}}=A_{2}^{i j} \frac{\delta h_{1}}{\delta u^{j}}
$$

where the Hamiltonian densities are the averaged Hamiltonians

$$
h_{2}=\frac{1}{2} \oint\left(\mathrm{u}^{3}+\mathrm{uu}_{x}^{2}+2 \nu \mathrm{u}^{2}\right) d \theta, \quad h_{1}=\frac{1}{2} \oint\left(\mathrm{u}^{2}+\mathrm{u}_{x}^{2}\right) d \theta .
$$

Moreover, the generating function for the Hamiltonian densities $h_{k}, k \geq 0$, is given by the coefficients of the expansion as $\lambda \rightarrow \infty$ of the differential

$$
\frac{\Omega_{\nu}(\lambda)}{\left\{\operatorname{Res}_{\lambda=-\nu} \frac{\left(\sigma_{1}(\lambda)\right)^{2}}{d \lambda}\right\}^{\frac{1}{2}}} \rightarrow-\left(\xi_{0}+\xi_{1} \frac{1}{\lambda}+\xi_{2} \frac{1}{\lambda^{2}}+\ldots\right) \frac{d \lambda}{\lambda^{2}} .
$$


In particular, the first Hamiltonians (modulo Casimirs) are

$$
h_{0}=2 \xi_{0}-\nu=\beta, \quad h_{1}=2 \xi_{1}+2 \nu \xi_{0}, \quad h_{2}=\frac{8}{3} \xi_{2}+6 \nu \xi_{1} .
$$

In the following we write the $\mathrm{CH}$-modulation equation in Hamiltonian form with respect to a nonlocal Hamiltonian operator of Mokhov-Ferapontov and Ferapontov type. Let $A_{3}^{i j}$ be the Hamiltonian operator of the form (35) associated to the metric $\frac{g_{i i}(\boldsymbol{u})}{4\left(\nu+u^{i}\right)^{2}}$ of constant curvature $c=1$, and let $A_{4}^{i j}$ be the Hamiltonian operator of the form (136) associated to the conformally flat metric $\frac{g_{i i}(\boldsymbol{u})}{8\left(\nu+u^{i}\right)^{3}}$ with affinors $\eta^{i}=C^{i}+\nu, i=1,2,3$.

The $\mathrm{CH}$ modulation equations (11) can also be written in a non-local Hamiltonian form

where

$$
\partial_{t} u^{i}=A_{3}^{i j} \frac{\delta h_{0}}{\delta u^{j}}=A_{4}^{i j} \frac{\delta h_{-1}}{\delta u^{j}}, \quad i=1,2,3,
$$

$$
h_{-1}=1-\frac{\nu}{\left\{\operatorname{Res}_{\lambda=-\nu} \frac{\left(\sigma_{1}(\lambda)\right)^{2}}{d \lambda}\right\}^{\frac{1}{2}}} .
$$

We remark that the Hamiltonian operators $A_{3}$ and $A_{4}$ can be obtained from the recursion $A_{3}=\mathcal{R}^{2} A_{1}$ and $A_{4}=\mathcal{R}^{3} A_{1}$ where $\mathcal{R}=A_{2} A_{1}^{-1}$ is the recursion operator and $A_{1}^{-1}$ denotes the inverse of $A_{1}$.

In the limit when two Riemann invariants coalesce, the Hamiltonian operators $A_{1}^{i j}$ and $A_{2}^{i j}$ reduce to the "dispersionless limit" of the Poisson operators of the $\mathrm{CH}$ equation

$$
\mathcal{P}_{1}=-\frac{d}{d x}, \quad \mathcal{P}_{2}=-2(\mathrm{u}+\nu) \frac{d}{d x}-\mathrm{u}_{x}
$$

respectively. In the same limit, the Hamiltonian operators $A_{3}^{i j}$ and $A_{4}^{i j}$ reduce to $\mathcal{P}_{2} \mathcal{P}_{1}^{-1} \mathcal{P}_{2}$ and $\mathcal{P}_{2} \mathcal{P}_{1}^{-1} \mathcal{P}_{2} \mathcal{P}_{1}^{-1} \mathcal{P}_{2}$, respectively.

\subsection{Integration of the Whitham equations}

In this subsection we show how to integrate the Whitham equations (11). All hydrodynamic systems satisfying (26) are integrable, via the generalized hodograph transform introduced by Tsarev [35. Indeed such systems (not necessarily with local Hamiltonian) possess an infinite number of commuting flows

$$
\partial_{t^{\prime}} u^{i}=w^{i}(\boldsymbol{u}) \partial_{x} u^{i}
$$


where the $w^{i}$ are solutions of the linear overdetermined system

$$
\frac{\partial_{u^{j}} w^{i}}{w^{i}-w^{j}}=\frac{\partial_{u^{j}} C^{i}}{C^{i}-C^{j}}, \quad i \neq j
$$

where $C^{i}=C^{i}\left(u^{1}, u^{2}, u^{3}\right), i=1,2,3$, are the speeds in (12). Then the solution $\boldsymbol{u}(x, t)=\left(u^{1}(x, t), u^{2}(x, t), u^{3}(x, t)\right)$ of the so-called hodograph transform

$$
x=-C^{i}(\boldsymbol{u}) t+w^{i}(\boldsymbol{u}) \quad i=1,2,3,
$$

satisfies the system (11). Conversely, any solution $\left(u^{1}(x, t), u^{2}(x, t), u^{3}(x, t)\right)$ of (11) can be obtained in this way. For monotone decreasing initial data $x=\left.f(u)\right|_{t=0}$, the general solution of the system (38) can be obtained following the work of Fei-Ran Tian [34] and the algebraic-geometric integration of Krichever 24]. For simplicity, we restrict ourselves to the case $\nu=0$.

Proposition 3.6 For $\nu=0$, and monotone increasing initial data $x=\left.f(u)\right|_{t=0}$, the solution of the system (38) is

$$
w^{i}(\boldsymbol{u})=q(\boldsymbol{u})+\left(C^{i}(\boldsymbol{u})-u^{1}-u^{2}-u^{3}\right) \frac{\partial q(\boldsymbol{u})}{\partial u^{i}},
$$

where the function $q=q(\boldsymbol{u})$ solves the linear over-determined system of EulerPoisson-Darboux type

$$
\begin{aligned}
& \partial_{u^{i}} q(\boldsymbol{u})-\partial_{u^{j}} q(\boldsymbol{u})=2\left(u^{i}-u^{j}\right) \partial_{u^{i}} \partial_{u^{j}} q(\boldsymbol{u}), \\
& q(u, u, u)=f(u) .
\end{aligned}
$$

The proof of the above statement follows from 33. Equation (41) can be integrated and the explicit expression of the function $q\left(u^{1}, u^{2}, u^{3}\right)$ is

$$
q\left(u^{1}, u^{2}, u^{3}\right)=\frac{1}{2 \sqrt{2 \pi}} \int_{-1}^{1} \int_{-1}^{1} \frac{f\left(\frac{1+\mu}{2} \frac{1+\eta}{2} u^{1}+\frac{1+\mu}{2} \frac{1-\eta}{2} u^{2}+\frac{1-\mu}{2} u^{3}\right)}{\sqrt{(1-\mu)\left(1-\eta^{2}\right)}} d \mu d \eta \text {. }
$$

\section{$4 \mathrm{CH}$ modulation equations versus $\mathrm{KdV}$ and re- ciprocal transformations}

In this section we compare $\mathrm{CH}$ and $\mathrm{KdV}$ modulation equations. We start recalling the reciprocal transformation which links the $\mathrm{CH}$ equation to the first negative $\mathrm{KdV}$ flow. We show that the $\mathrm{CH}$ modulation equations (11) are transformed to the modulation equations of the first negative KdV flow by the averaged reciprocal transformation. Finally we compare the averaged Hamiltonian operators of the two systems. 


\subsection{A reciprocal transformation between Camassa Holm equa- tion and the first negative flow of $\mathrm{KdV}$}

In this subsection we summarize the relation between the Camassa Holm equation and the first negative flow of KdV hierarchy [21. The (associated) Camassa-Holm equation is transformed into the first negative $\mathrm{KdV}$ flow by a reciprocal transformation. In the following, to distinguish between $\mathrm{CH}$ and $\mathrm{KdV}$, we use $(x, t)$ for Camassa-Holm variables and $\left(y, \tau_{-}\right)$for the $\mathrm{KdV}$ variables.

The change of dependent variable $\rho^{2}=m+\nu$ transforms the Camassa-Holm equation

$$
m_{t}=-2 m \mathrm{u}_{x}-\mathrm{u}_{x}-2 \nu \mathrm{u}_{x}, \quad m=\mathrm{u}-\mathrm{u}_{x x} .
$$

into the associated Camassa-Holm equation

$$
\left\{\begin{array}{l}
\rho_{t}=-(\mathrm{u} \rho)_{x} \\
\rho^{2}=\mathrm{u}-\mathrm{u}_{x x}+\nu,
\end{array}\right.
$$

which, via the reciprocal transformation introduced by Fuchssteiner [21],

$$
\left\{\begin{array}{l}
d y=\rho d x-\mathrm{u} \rho d t \\
d \tau_{-}=d t
\end{array}\right.
$$

is finally transformed into

$$
\left\{\begin{array}{l}
\mathrm{u}=\rho^{2}-\nu-\rho_{y \tau_{-}}+\frac{\rho_{\tau_{-}} \rho_{y}}{\rho} \\
\left(\frac{1}{\rho}\right)_{\tau_{-}}=2 \rho \rho_{y}-\left(\rho(\log \rho)_{y \tau_{-}}\right)_{y} .
\end{array}\right.
$$

The transformation (43) is a reciprocal transformation because the one-form $\rho d x-$ u $\rho d t$ is closed with respect to the $\mathrm{CH}$ flow.

The equation (44) is equivalent to the first negative flow of the KdV hierarchy

$$
\left(\partial_{y}^{2}+2 U+U_{y} \partial_{y}^{-1}\right) U_{\tau_{-}}=0
$$

under the condition $U_{\tau_{-}}=-2 \rho_{y}$. Equation (45) may be re-expressed as

$$
\left\{\begin{array}{l}
U=\frac{\rho_{y}^{2}-2 \rho \rho_{y y}-1}{2 \rho^{2}} \\
\left(\frac{\rho_{y}^{2}-2 \rho \rho_{y y}-1}{4 \rho^{2}}\right)_{\tau_{-}}=-\rho_{y} .
\end{array}\right.
$$


Finally, we observe that $\int \rho(x, t) d x$ is a Casimir of the second Hamiltonian operator of the Camassa-Holm equation, $P_{2}=m \partial_{x}+\partial_{x} m+2 \nu \partial_{x}$.

The $2 \pi$-periodic solutions of the first negative $\mathrm{KdV}$ flow (45), $U(\Theta), \Theta=\mathcal{K} y-\Omega \tau$, satisfy

$$
d \Theta=\frac{\mathcal{K} d U}{\sqrt{-U^{3}+\alpha U^{2}-\beta U+\gamma}} .
$$

We may express such solutions also in the form $\rho(\Theta)$ or $u(\Theta)$ and we easily get

$$
\left\{\begin{array}{l}
\mathrm{u}_{\Theta}^{2}=\frac{1}{C^{2} \mathcal{K}^{2}}(\mathrm{u}-c)\left(\mathrm{u}^{3}-(c-2 \nu) \mathrm{u}^{2}+2 B \mathrm{u}-2 A\right) . \\
\rho(\Theta)=-\frac{C}{\mathrm{u}(\Theta)-c} \\
U(\Theta)=-\frac{2}{\mathrm{u}(\Theta)-c}
\end{array}\right.
$$

where $C=\Omega / \mathcal{K}$ and $A, B, c$ are the constants defined in (9) which satisfy

$$
B c+\nu c^{2}-A=C^{2}, \quad \alpha=-\frac{c^{2}+2 B+4 \nu c}{C^{2}} .
$$

The periodic solutions $u(\theta), \rho(\theta), \theta=k x-\omega t$ of the (associated) Camassa-Holm equation, satisfy

$$
d \theta=\frac{k(\mathrm{u}-c) d \mathrm{u}}{\sqrt{(\mathrm{u}-c)\left(\mathrm{u}^{3}-(c-2 \nu) \mathrm{u}^{2}+2 B \mathrm{u}-2 A\right)}} .
$$

We observe that the reciprocal transformation sends $2 \pi$-periodic solutions in $\Theta$ into $2 \pi$-periodic solution in $\theta$ (and vice versa). Indeed, let $T$ be the period of $\mathrm{u}(\theta)$, then

$$
T=\frac{k}{\mathcal{K}} \int_{0}^{2 \pi} \frac{c-\mathrm{u}}{C} d \Theta=k \oint \frac{(\mathrm{u}-c) d \mathrm{u}}{\sqrt{(\mathrm{u}-c)\left(\mathrm{u}^{3}-(c-2 \nu) \mathrm{u}^{2}+2 B \mathrm{u}-2 A\right)}}=2 \pi .
$$

It is then natural to expect that the average of the reciprocal transformation connects the $\mathrm{CH}$ modulation equations to the modulation equations of the first negative $\mathrm{KdV}$ flow.

\subsection{The modulation equations of the negative $\mathrm{KdV}$ flow}

In this subsection, we compute the modulation equations of the KdV negative flow in the Riemann invariant coordinates used in the literature, namely the branch points 
$\beta^{1}, \beta^{2}, \beta^{3}$, of the odd elliptic curve 37]

$$
w^{2}=\left(\eta-\beta^{1}\right)\left(\eta-\beta^{2}\right)\left(\eta-\beta^{3}\right),
$$

where

$$
\beta^{1}+\beta^{2}+\beta^{3}=\alpha,
$$

with $\alpha$ defined in (47). It turns out from (49) that

$$
\beta^{i}=\frac{1}{u^{i}+\nu}>0, \quad i=1,2,3,
$$

where $u^{i}$ are the $\mathrm{CH}$ Riemann invariants defined in theorem 3.1. On the Riemann surface (50) we define the second kind normalized differential

$$
d p(\eta)=\frac{\eta+\alpha_{1}}{\sqrt{\left(\eta-\beta^{1}\right)\left(\eta-\beta^{2}\right)\left(\eta-\beta^{3}\right)}} d \eta
$$

where $\alpha_{1}$ is uniquely determined by the normalization condition

$$
\oint_{a} d p(\eta)=0
$$

In the $\mathrm{KdV}$ literature $d p(\eta)$ is known as quasi-momentum. Now we can compute the velocities of the modulation equations of the negative $\mathrm{KdV}$ flow

Proposition 4.1 The one phase Whitham equations of the first negative KdV flow are

$$
\partial_{\tau_{-}} \beta^{i}+v^{i}(\beta) \partial_{y} \beta^{i}=0
$$

where

$$
v^{i}(\beta):=\frac{\partial_{i} \Omega(\beta)}{\partial_{i} \mathcal{K}(\beta)}=\frac{2}{\sqrt{\beta^{1} \beta^{2} \beta^{3}}}\left(1-\frac{\prod_{j \neq i}\left(\beta^{i}-\beta^{j}\right)}{\beta^{i}\left(\beta^{i}+\alpha_{1}\right)}\right),
$$

with $\alpha_{1}$ as in (52). In the above relations $\Omega$ and $\mathcal{K}$ are the frequency and wavenumber of the one-phase KdV negative flow.

The proof of the proposition is as follows. From (47) and (48), we immediately get $\mathcal{K}=2 \pi \mathcal{J}_{0}^{-1}$ and $\Omega=C \mathcal{K}=4 \pi \mathcal{J}_{0}^{-1}\left(\beta^{1} \beta^{2} \beta^{3}\right)^{-1 / 2}$, where

$$
\mathcal{J}_{0}=\oint \frac{d \lambda}{\sqrt{\left(\lambda-\beta^{1}\right)\left(\lambda-\beta^{2}\right)\left(\lambda-\beta^{3}\right)}} .
$$


Then the expressions for the velocities (154) are computed from the definition using the following variational formula

$$
\frac{\partial \mathcal{J}_{0}}{\partial \beta^{i}}=\frac{1}{2} \mathcal{J}_{0} \frac{\beta^{i}+\alpha_{1}}{\prod_{j \neq i}\left(\beta^{i}-\beta^{j}\right)}, \quad i=1,2,3 .
$$

Remark 4.2 In the limit $\beta^{2}=\beta^{3}$, the equations (53) converge to $\beta_{\tau_{-}}^{1}=-2\left(\beta^{1}\right)^{-\frac{3}{2}} \beta_{y}^{1}$, which is the dispersionless limit of the first negative $K d V$ flow.

Next, we write the negative KdV equations in Hamiltonian form. In the $\beta$ s' coordinates, the flat metric associated to the first local KdV-Whitham Hamiltonian operator is 13 ]

$$
g_{i i}^{K d V}(\boldsymbol{\beta})=\operatorname{Res}_{\eta=\beta^{i}}\left\{\frac{(d p(\eta))^{2}}{d \eta}\right\}, \quad i=1,2,3,
$$

where the differential $d p$ has been defined in (152).

Remark 4.3 The flat metrics $g_{i i}^{K d V}(\boldsymbol{\beta})$ and $g_{i i}^{K d V}(\boldsymbol{\beta}) / \beta^{i}$ can be related to a Frobenius manifold defined on the moduli space of elliptic curves $w^{2}=\left(\eta-\beta^{1}\right)\left(\eta-\beta^{2}\right)(\eta-$ $\left.\beta^{3}\right)$ [14].

Let $J_{1}$ and $J_{2}$ be the local KdV Hamiltonian operators of the form (34) associated to the flat metrics $\frac{1}{8} g_{i i}^{K d V}(\boldsymbol{\beta})$ and $\frac{g_{i i}^{K d V}(\boldsymbol{\beta})}{4 \beta^{i}}$. Let $J_{3}$ be the nonlocal Hamiltonian operator of the form (35) associated to the metric $\frac{g_{i i}^{K d V}(\boldsymbol{\beta})}{2\left(\beta^{i}\right)^{2}}$ of constant curvature $R_{i j}^{i j}=-\frac{1}{2}$. Let $J_{4}$ be the nonlocal Hamiltonian operator of the form (36) associated to the conformally flat metric $\frac{g_{i i}^{K d V}(\boldsymbol{\beta})}{\left(\beta^{i}\right)^{3}}$ with Riemannian curvature $R_{i j}^{i j}=-\frac{1}{8}\left(w_{+}^{i}+\right.$ $\left.w_{+}^{j}\right)$, where

$$
w_{+}^{i}=\left(\beta^{1}+\beta^{2}+\beta^{3}+\frac{2 \prod_{j \neq i}\left(\beta^{i}-\beta^{j}\right)}{\beta^{i}+\alpha_{1}}\right),
$$

with $\alpha_{1}$ defined in (52). The $w_{+}^{i}, i=1, \ldots, 3$, are the velocities (originally obtained by Whitham [37]) of the usual positive $\mathrm{KdV}$ modulated flow

$$
\frac{\partial \beta^{i}}{\partial \tau_{+}}+w_{+}^{i}(\boldsymbol{\beta}) \frac{\partial \beta^{i}}{\partial y}=0
$$


Remark 4.4 The Hamiltonian operator $J_{1}$ corresponds to the average over a onedimensional torus of the Gardner-Zakharov KdV Hamiltonian structure $\mathcal{P}_{1}=8 \partial_{y}$ while the Hamiltonian operator $J_{2}$ corresponds to the average over a one-dimensional torus of the Lenard-Magri local Hamiltonian structure $\mathcal{P}_{2}=2 \partial_{y y y}+2 U \partial_{y}+2 \partial_{y} U$. We use this unusual normalization in order to be consistent with the normalization of the $C H$ equation. Defining the recursive operator $\mathcal{R}=\mathcal{P}_{2}\left(\mathcal{P}_{1}\right)^{-1}$, we obtain the family of non-local Hamiltonian operators $\mathcal{P}_{k+1}=\mathcal{R P}_{k}, k \geq 1$. The averaged $K d V$ non-local Hamiltonian operators $J_{3}$ and $J_{4}$ corresponds to the average over a onedimensional torus of the non-local Hamiltonian operators $\mathcal{P}_{3}$ and $\mathcal{P}_{4}$, respectively.

The modulation equations of the negative $\mathrm{KdV}$ flow can be written in Hamiltonian form with Hamiltonian operator $J_{1}$ and Hamiltonian density $\mathcal{H}_{0}$, which is the average over the one dimensional torus of the Casimir generating the $\mathrm{KdV}$ negative flow, namely

$$
\mathcal{H}_{0}=\oint \frac{d \Theta}{\rho(\Theta)}
$$

Lemma 4.5 [14] In the $\beta s^{\prime}$ coordinates

$$
\mathcal{H}_{0}=i p(0)
$$

where $p(\eta)$ is the Abelian integral of the quasi-momentum $d p(\eta)$ defined in (52).

To prove the lemma, we compute the integral in (58) in the $\beta \mathrm{s}^{\prime}$ coordinates obtaining

$$
\mathcal{H}_{0}=\oint \frac{d \Theta}{\rho(\Theta)}=-\sqrt{\beta^{1} \beta^{2} \beta^{3}} \alpha_{0}=-\left.i \frac{\left(\Lambda_{0}(\eta)\right)}{2 d \xi}\right|_{\xi=0}, \quad \eta=\frac{1}{\xi^{2}},
$$

where

$$
\Lambda_{0}(\eta)=\sqrt{-\beta^{1} \beta^{2} \beta^{3}} \frac{\frac{1}{\eta}+\alpha_{0}}{\sqrt{\left(\eta-\beta^{1}\right)\left(\eta-\beta^{2}\right)\left(\eta-\beta^{3}\right)}} d \eta
$$

$\Lambda_{0}(\eta)$ is a normalized third kind differential with first order poles at the points $O^{ \pm}=\left(0, \pm \sqrt{-\beta^{1} \beta^{2} \beta^{3}}\right)$ with residue \pm 1 , respectively. The constant $\alpha_{0}$ in (59) is uniquely determined by the condition $\oint_{a} \Lambda_{0}(\eta)=0$. From the Riemann bilinear relation, it is finally immediate to verify that

$$
\mathcal{H}_{0}=-\left.i \frac{\left(\Lambda_{0}(\eta)\right)}{2 d \xi}\right|_{\xi=0}=i p(0), \quad \eta=\frac{1}{\xi^{2}},
$$


where $p(\eta)$ is the Abelian integral of the quasi-momentum $d p(\eta)$ defined in (52). Finally the following result holds.

Lemma 4.6 The first negative KdV averaged flow (53) can be written in the Hamiltonian form

$$
\beta_{\tau_{-}}^{i}=J_{1}^{i j} \frac{\delta \mathcal{H}_{0}}{\delta \beta^{j}}=J_{2}^{i j} \frac{\delta \mathcal{H}_{-1}}{\delta \beta^{j}}=J_{3}^{i j} \frac{\delta \mathcal{H}_{-2}}{\delta \beta^{j}}=J_{4}^{i j} \frac{\delta \mathcal{H}_{-3}}{\delta \beta^{j}}
$$

where $\mathcal{H}_{0}$ is the Casimir of $J_{2}$ defined in (58) and the Hamiltonian densities are determined by the recursion scheme

$$
J_{1}^{i j} \frac{\delta \mathcal{H}_{-s}}{\delta \beta^{j}}=J_{2}^{i j} \frac{\delta \mathcal{H}_{-s-1}}{\delta \beta^{j}}, \quad s \geq 0 .
$$

The Hamiltonian $\mathcal{H}_{-s}, s \geq 1$, are generated by the expansion for $\eta \rightarrow 0$ of the quasi-momentum $d p(\eta)$. Indeed,

$$
i \int_{(\eta,-w)}^{(\eta, w)} d p(\xi)=\frac{1}{2}\left(\mathcal{H}_{0}+\eta \mathcal{H}_{-1}+\eta^{2} \mathcal{H}_{-2}+\frac{3}{4} \eta^{3} \mathcal{H}_{-3}+\ldots\right), \quad \eta \rightarrow 0 .
$$

\section{3 $\mathrm{CH}$ versus $\mathrm{KdV}$ modulation equations}

In the previous section we computed the modulation equations of the first negative $\mathrm{KdV}$ flow. In this subsection, we compute the average of the reciprocal transformation defined in section 4.1 and we show that the negative $\mathrm{KdV}$ modulation equations are transformed to the $\mathrm{CH}$ modulation equations. Finally, both KdV-Whitham and $\mathrm{CH}-$ Whitham systems are Hamiltonian systems, so we end the section investigating how the reciprocal transformation acts on the Hamiltonian structures of the two systems.

To compare $\mathrm{KdV}$ and $\mathrm{CH}$, we first need to reduce the even spectral curve of $\mathrm{CH}$ to the odd spectral curve of $\mathrm{KdV}$. The natural change of coordinates $(\lambda, y) \rightarrow(\eta, w)$

$$
\eta=\frac{1}{\lambda+\nu}, \quad w^{2}=-\frac{y^{2}}{(\lambda+\nu)^{4} \prod_{i=1}^{3}\left(\nu+u^{i}\right)}
$$

maps the even spectral curve $y^{2}=(\lambda+\nu)\left(\lambda-u^{1}\right)\left(\lambda-u^{2}\right)\left(\lambda-u^{3}\right)$ to the odd $\mathrm{KdV}$ spectral curve

$$
w^{2}=\left(\eta-\beta^{i}\right)\left(\eta-\beta^{2}\right)\left(\eta-\beta^{3}\right), \quad \beta^{i}=\frac{1}{\nu+u^{i}} .
$$


The differentials $\Omega_{\nu}(\lambda)$ and $\sigma_{1}(\lambda)$ defined in (24) and (15) transform to

$$
\begin{aligned}
\Omega_{\nu}(\lambda) & \rightarrow \frac{1}{2} d p(\eta), \\
\sigma_{1}(\lambda) & \rightarrow \Lambda_{0}(\eta),
\end{aligned}
$$

with $d p(\eta)$ as in (52) and $\Lambda_{0}(\eta)$ as in (59). It follows from (63) and (64) that the change of coordinates $\beta^{i}=1 /\left(\nu+u^{i}\right)$ transforms the speeds $C^{i}(\boldsymbol{u})$ defined in (25) to

$$
\tilde{C}^{i}(\boldsymbol{\beta})=\frac{1}{\beta^{1}}+\frac{1}{\beta^{2}}+\frac{1}{\beta^{3}}-\nu+2 \frac{\alpha_{0} \prod_{j \neq i, j=1}^{3}\left(\beta^{i}-\beta^{j}\right)}{\beta^{i}\left(\beta^{i}+\alpha_{1}\right)} .
$$

Now we show that the averaged reciprocal transformation maps the modulation equations of the $\mathrm{KdV}$ negative flow to the $\mathrm{CH}$ modulation equations. Indeed averaging over a period the inverse of (43)

$$
d x=1 / \rho d y+\mathrm{u} d \tau_{-}, \quad d t=d \tau_{-},
$$

we get the averaged reciprocal transformation

$$
d x=d y \oint \frac{d \Theta}{\rho(\Theta)}+d \tau_{-} \oint \mathrm{u}(\Theta) d \Theta \quad d t=d \tau_{-} .
$$

Proposition 4.7 The averaged reciprocal transformation (666) takes the form

$$
d x=\mathcal{H}_{0} d y+\mathcal{N} d \tau_{-}, \quad d t=d \tau_{-},
$$

where $\mathcal{H}_{0}$ is the Casimir defined in (58) and

$$
\mathcal{N}=\frac{1}{\beta^{1}}+\frac{1}{\beta^{2}}+\frac{1}{\beta^{3}}-\nu+2 \alpha_{0}=\frac{1}{2}\left(\nabla \mathcal{H}_{0}\right)^{2}-\nu,
$$

where $\left(\nabla \mathcal{H}_{0}\right)^{2}=\sum_{i}\left(g_{i i}^{K d V}\right)^{-1}\left(\partial_{\beta^{i}} \mathcal{H}_{0}\right)^{2}$. Finally (67) is a reciprocal transformation for the $K d V$-Whitham negative flow.

To prove the proposition we observed that the one form (67) is closed by (68). The proof of (68) follows from the identity $\mathcal{H}_{0}=i p(0)$, and the variational formula $\partial_{\beta^{i}} p(0)=\frac{1}{4} d p\left(\beta^{i}\right) \Lambda_{0}\left(\beta^{i}\right)$.

Next we show that the modulation equations of the first negative KdV flow are mapped by the reciprocal transformation (67) to the $\mathrm{CH}$ modulation equations. 
Proposition 4.8 The reciprocal transformation $d x=\mathcal{H}_{0} d y+\mathcal{N} d \tau_{-}$, $d t=d \tau_{-}$, where $\mathcal{H}_{0}$ and $\mathcal{N}$ are as in (58) and (68) respectively, transforms the modulation equations (53) of the first negative $K d V$ flow

$$
\partial_{\tau_{-}} \beta^{i}+v^{i}(\boldsymbol{\beta}) \partial_{y} \beta^{i}=0
$$

where $v^{i}(\boldsymbol{\beta}), i=1, \ldots, 3$ are as in (54) to the CH modulation equations

$$
\partial_{t} \beta^{i}+\tilde{C}^{i}(\boldsymbol{\beta}) \partial_{x} \beta^{i}=0
$$

where the CH velocities are defined in (65). Viceversa, the inverse reciprocal transformation $d y=\frac{1}{\mathcal{H}_{0}} d x-\frac{\mathcal{N}}{\mathcal{H}_{0}} d t$ transforms (70) into (69).

Indeed plugging (67) into (69) we get $\partial_{t} \beta^{i}+V^{i}(\boldsymbol{\beta}) \partial_{x} \beta^{i}=0$, where

$$
V^{i}(\boldsymbol{\beta})=v^{i}(\boldsymbol{\beta}) \mathcal{H}_{0}+\mathcal{N}=\tilde{C}^{i}(\boldsymbol{\beta}) .
$$

To compare the $\mathrm{CH}$ and $\mathrm{KdV}$ Hamiltonian structures, we express the $\mathrm{CH}$ Whitham Hamiltonian operators introduced in subsection 3.1 in the $\beta$ s' coordinates. In these coordinates, using (60), (62) and (63), the $\mathrm{CH}$ flat metric $g_{i i}(\boldsymbol{u})$ defined in (27) takes the form

$$
\tilde{g}_{i i}(\boldsymbol{\beta})=\frac{-1}{\left(\beta^{i}\right)^{3}} \frac{\operatorname{Res}_{\eta=\beta^{i}}\left\{\frac{(d p(\eta))^{2}}{d \eta}\right\}}{p(0)^{2} / 4}=\frac{g_{i i}^{K d V}(\boldsymbol{\beta})}{\left(\beta^{i}\right)^{3} \mathcal{H}_{0}^{2}}, \quad i=1,2,3 .
$$

The other metrics defined in (28) transform, respectively, to

$$
\frac{g_{i i}^{K d V}(\boldsymbol{\beta})}{2\left(\beta^{i}\right)^{2} \mathcal{H}_{0}^{2}}, \quad \frac{g_{i i}^{K d V}(\boldsymbol{\beta})}{4 \beta^{i} \mathcal{H}_{0}^{2}}, \quad \frac{g_{i i}^{K d V}(\boldsymbol{\beta})}{8 \mathcal{H}_{0}^{2}},
$$

and are, respectively, flat, of constant curvature and conformally flat.

Let $\tilde{A}_{1}$ and $\tilde{A}_{2}$ be the local Hamiltonian operators associated to the $\mathrm{CH}$ flat metrics $\tilde{g}_{i i}(\boldsymbol{\beta})$ and $\frac{1}{2} \tilde{g}_{i i}(\boldsymbol{\beta}) \beta^{i}$, respectively. Moreover, let $\tilde{A}_{3}$ and $\tilde{A}_{4}$ be the non-local Hamiltonian operators associated to the $\mathrm{CH}$ constant curvature and conformally flat metrics, $\frac{1}{4} \tilde{g}_{i i}(\boldsymbol{\beta})\left(\beta^{i}\right)^{2}$ and $\frac{1}{8} \tilde{g}_{i i}(\boldsymbol{\beta})\left(\beta^{i}\right)^{3}$, respectively. Then, in the $\beta$ s' coordinates the $\mathrm{CH}$ modulation equations in Hamiltonian form are

$$
\beta_{t}^{i}=\tilde{A}_{1}^{i j} \frac{\delta \tilde{h}_{2}}{\delta \beta^{j}}=\tilde{A}_{2}^{i j} \frac{\delta \tilde{h}_{1}}{\delta \beta^{j}}=\tilde{A}_{3}^{i j} \frac{\delta \tilde{h}_{0}}{\delta \beta^{j}}=\tilde{A}_{4}^{i j} \frac{\delta \tilde{h}_{-1}}{\delta \beta^{j}}
$$


where the Hamiltonians $\tilde{h}_{j}, i=-1, \ldots, 2$, are the averaged conservation laws introduced in subsection 3.1 expressed in the $\beta \mathrm{s}^{\prime}$ coordinates. Indeed, following (62), the positive $\mathrm{CH}$ Hamiltonians are obtained from the coefficients of the expansion for $\eta \rightarrow 0$ of the differential $\frac{d p(\eta)}{\mathcal{H}_{0}}$ and take the form

$$
\tilde{h}_{-1}=1-\frac{\nu}{\mathcal{H}_{0}}, \quad \tilde{h}_{0}=\frac{\mathcal{H}_{-1}}{\mathcal{H}_{0}}-\nu, \quad \tilde{h}_{j}=\frac{\mathcal{H}_{-j-1}}{\mathcal{H}_{0}}-\nu \frac{\mathcal{H}_{-j}}{\mathcal{H}_{0}}, \quad j \geq 1,
$$

where the $\mathcal{H}_{-k}$ are defined in (62). The following theorem by Ferapontov and Pavlov describes the action of a reciprocal transformation for an Hamiltonian hydrodynamic equation and we use it to clarify the relation between the Hamiltonian structures of the averaged $\mathrm{KdV}$ and $\mathrm{CH}$ Hamiltonian structures.

Theorem 4.9[18] Let $\beta_{\tau}^{i}=J^{i j} \frac{\partial h}{\partial \beta^{j}}$ be an Hamiltonian system associated to the local operator $J^{i j}=g^{i i} \delta_{i}^{j} \frac{d}{d y}-g^{i i} \Gamma_{i k}^{j} \beta_{y}^{k}$. Then, under the action of the reciprocal transformation $d x=A d y+B d \tau, d t=d \tau$, where $d(A d y+B d \tau)=0$, the transformed system is Hamiltonian $\beta_{t}^{i}=\tilde{J}^{i j} \frac{\partial \tilde{h}}{\partial \beta^{j}}$, with nonlocal operator

$$
\tilde{J}^{i j}=\tilde{g}^{i i} \delta^{i j} \frac{d}{d x}-\tilde{g}^{i i} \tilde{\Gamma}_{i k}^{j} \beta_{x}^{k}+\tilde{w}^{i} \beta_{x}^{i}\left(\frac{d}{d x}\right)^{-1} \beta_{x}^{j}+\beta_{x}^{i}\left(\frac{d}{d x}\right)^{-1} \tilde{w}^{j} \beta_{x}^{j}
$$

and hamiltonian density $\tilde{h}=h / A$. The transformed metric is $\tilde{g}_{i i}=g_{i i} / A^{2}, \tilde{\Gamma}$ is the Levi-Civita connection and

$$
\tilde{w}^{i}=\nabla^{i} \nabla_{i} A \cdot A-\frac{1}{2}(\nabla A)^{2}=g^{i i}\left(\partial_{i}^{2} A-\sum_{j} \Gamma_{i i}^{j} \partial_{j} A\right) A-\frac{1}{2} \sum_{j} g^{j j}\left(\partial_{j} A\right)^{2} .
$$

Moreover, the transformed metric is conformally flat with curvature tensor

$$
\tilde{R}_{i j}^{i j}=\tilde{w}^{i}+\tilde{w}^{j}
$$

By the above theorem and by inspection of (61) and (73), where the metrics have been defined in (171), (172) and (156), we conclude that the local KdV-Whitham Poisson operators, $J_{1}$ and $J_{2}$, are mapped to the nonlocal CH-Whitham Poisson operators $\tilde{A}_{4}$ and $\tilde{A}_{3}$, respectively, by the reciprocal transformation $d x=\mathcal{H}_{0} d y+$ $\mathcal{N} d \tau_{-}, d t=d \tau_{-}$. 
Remark 4.10 The application of theorem 4.9 deserves a special attention in the case $\nu \neq 0$ for the computation of the Hamiltonian densities $\tilde{h}_{k}$. Indeed neither the $K d V$-Hamiltonian operators $J_{i}$ nor the averaged negative $K d V$ Hamiltonian densities $\mathcal{H}_{-s}, s=0,1,2,3$, depend on the parameter $\nu$ while the $C H$ averaged Hamiltonian densities $\tilde{h}_{s}, s=-1,0,1,2$, in (74) do. To solve this apparent contradiction, we recall that the Hamiltonian densities are defined modulo Casimirs.

In particular, the Casimir $-\nu \mathcal{H}_{0}$ of the second KdV-Whitham Hamiltonian operator is transformed to the CH Hamiltonian density $-\nu$ which generates the constant flow term associated to $\tilde{A}_{3}$. Similarly, the term $-\nu$ is mapped by the reciprocal transformation to the $\mathrm{CH}$ Hamiltonian density $-\nu / \mathcal{H}_{0}$ which generates the constant flow term associated to $\tilde{A}_{4}$.

Applying theorem 4.9 to the local $\mathrm{CH}-$ Whitham Poisson operators, we prove that the local Hamiltonian $\mathrm{CH}$ operators $\tilde{A}_{1}$ and $\tilde{A}_{2}$, are mapped to the nonlocal $\mathrm{KdV}$-Whitham Poisson operators $J_{4}$ and $J_{3}$, respectively, by the reciprocal transformation $d y=1 / \mathcal{H}_{0} d x-\mathcal{N} / \mathcal{H}_{0} d t$. By the same theorem 4.9, the corresponding $\mathrm{KdV}$ Hamiltonian densities are related to the $\mathrm{CH}$ ones by

$$
\tilde{h}_{2} \mathcal{H}_{0}=\mathcal{H}_{-3}, \quad \tilde{h}_{1} \mathcal{H}_{0}=\mathcal{H}_{-2},
$$

where the above identities again hold modulo Casimirs of the corresponding KdV Hamiltonian operators. We summarize the results of this section in the following.

Theorem 4.11 The reciprocal transformation $d x=\mathcal{H}_{0} d y+\mathcal{N} d \tau_{-}$, $d t=d \tau_{-}$, maps the KdV local Hamiltonian operators $J_{1}, J_{2}$ and the corresponding Hamiltonian densities $\mathcal{H}_{0}, \mathcal{H}_{-1}$ to

$$
\begin{aligned}
J_{1}^{i k} \frac{\delta \mathcal{H}_{0}}{\delta \beta^{k}} \longrightarrow \tilde{A}_{4}^{i k} \frac{\delta \tilde{h}_{-1}}{\delta \beta^{k}} \\
J_{2}^{i k} \frac{\delta \mathcal{H}_{-1}}{\delta \beta^{k}} \longrightarrow \tilde{A}_{3}^{i k} \frac{\delta \tilde{h}_{0}}{\delta \beta^{k}}
\end{aligned}
$$

where $\tilde{A}_{4}$ is the CH nonlocal Hamiltonian operator associated to the covariant conformally flat metric $\frac{g_{i i}^{K d V}(\boldsymbol{\beta})}{8 \mathcal{H}_{0}^{2}}$ and $\tilde{h}_{-1}=1-\nu / \mathcal{H}_{0}$ the corresponding CH Hamiltonian density $\left(\tilde{h}_{-1} \mathcal{H}_{0}=\mathcal{H}_{0}\right.$ modulo Casimir of $\left.J_{1}\right)$. The Hamiltonian operator $\tilde{A}_{3}$ is the $C H$ nonlocal Hamiltonian operator associated to the constant curvature metric $\frac{g_{i i}^{K d V}(\boldsymbol{\beta})}{4 \mathcal{H}_{0}^{2} \beta^{i}}$ and $\tilde{h}_{0}=-\nu+\frac{\mathcal{H}_{-1}}{\mathcal{H}_{0}}$ the corresponding CH Hamiltonian density $\tilde{h}_{0} \mathcal{H}_{0}=\mathcal{H}_{-1}$ modulo a Casimir of $\left.J_{2}\right)$. 
The reciprocal transformation $d y=1 / \mathcal{H}_{0} d x-\mathcal{N} / \mathcal{H}_{0} d t, d \tau_{-}=d t$ maps the local CH Hamiltonian operators $\tilde{A}_{1}, \tilde{A}_{2}$, and corresponding Hamiltonian densities $\tilde{h}_{2}=$ $\frac{\mathcal{H}_{-3}}{\mathcal{H}_{0}}-\nu \frac{\mathcal{H}_{-2}}{\mathcal{H}_{0}}, \tilde{h}_{1}=\frac{\mathcal{H}_{-2}}{\mathcal{H}_{0}}-\nu \frac{\mathcal{H}_{-1}}{\mathcal{H}_{0}}$ to

$$
\begin{aligned}
& \tilde{A}_{1}^{i k} \frac{\delta \tilde{h}_{2}}{\delta \beta^{k}} \longrightarrow J_{4}^{i k} \frac{\delta \mathcal{H}_{-3}}{\delta \beta^{k}} \\
& \tilde{A}_{2}^{i k} \frac{\delta \tilde{h}_{1}}{\delta \beta^{k}} \longrightarrow J_{3}^{i k} \frac{\delta \mathcal{H}_{-2}}{\delta \beta^{k}}
\end{aligned}
$$

where the nonlocal KdV Hamiltonian operator $J_{4}$ is associated to the covariant conformally flat metric $\frac{g_{i i}^{K d V}(\boldsymbol{\beta})}{\left(\beta^{i}\right)^{3}}$ and $\tilde{h}_{2} \mathcal{H}_{0}=\mathcal{H}_{-3}$ modulo a Casimir of $J_{4}$. The nonlocal KdV Hamiltonian operator $J_{3}$ is associated to the covariant constant curvature metric $\frac{g_{i i}^{K d V}(\boldsymbol{\beta})}{2\left(\beta^{i}\right)^{2}}$ and $\tilde{h}_{1} \mathcal{H}_{0}=\mathcal{H}_{-2}$ modulo a Casimir of $J_{3}$.

We conclude this section by illustrating with a table all the reciprocal transformations between the various Hamiltonian operators.

Acknoledgments We express our gratitude to A. Maltsev for the many useful discussions and for pointing out to us the Lagrangian averaging method for the Camassa-Holm equations. We also thank B. Dubrovin, G. Falqui and D. Holm for the useful discussions and comments of the manuscript. This work has been partially supported by the GNFM-INdAM Project "Onde nonlineari, struttura tau e geometria delle varietà invarianti: il caso della gerarchia di Camassa-Holm" and by the European Science Foundation Programme MISGAM (Method of Integrable Systems, Geometry and Applied Mathematics).

\section{References}

[1] Abenda; S., Fedorov, Yu. On the weak Kowalevski-Painlevé property for hyperelliptically separable systems. Acta Appl. Math., 60 (2), ( 2000): 137-178.

[2] Ablowitz, M.J.; Kaup, D.J.; Newell, A.C.; Segur, H. The inverse scattering transform-Fourier analysis for nonlinear problems Studies in Appl. Math $\mathbf{5 3}$ (1974), 249-315. 


\begin{tabular}{|c|c|c|c|c|}
\hline $\begin{array}{l}\text { KdV Poisson } \\
\text { tensor }\end{array}$ & $\mathcal{P}_{1}$ & $\mathcal{P}_{2}$ & $\mathcal{R}^{2} \mathcal{P}_{1}$ & $\mathcal{R}^{3} \mathcal{P}_{1}$ \\
\hline $\begin{array}{l}\text { averaged } \mathrm{KdV} \\
\text { Poisson tensor }\end{array}$ & $J_{1}$ & $J_{2}$ & $J_{3}$ & $J_{4}$ \\
\hline $\mathrm{KdV}$ metric & $\frac{1}{8} g_{i i}^{K d V}(\boldsymbol{\beta})$ & $\frac{g_{i i}^{K d V}(\boldsymbol{\beta})}{4 \beta^{i}}$ & $\frac{g_{i i}^{K d V}(\boldsymbol{\beta})}{2\left(\beta^{i}\right)^{2}}$ & $\frac{g_{i i}^{K d V}(\boldsymbol{\beta})}{\left(\beta^{i}\right)^{3}}$ \\
\hline Curvature tensor $R_{i j}^{i j}$ & 0 & 0 & $-1 / 2$ & $-\left(w_{+}^{i}+w_{+}^{j}\right) / 8$ \\
\hline $\begin{array}{c}\text { averaged KdV } \\
\text { Hamiltonian }\end{array}$ & $\mathcal{H}_{0}-\nu$ & $\mathcal{H}_{-1}-\nu \mathcal{H}_{0}$ & $\mathcal{H}_{-2}-\nu \mathcal{H}_{-1}$ & $\mathcal{H}_{-3}-\nu \mathcal{H}_{-2}$ \\
\hline $\begin{array}{c}\text { Reciprocal } \\
\text { transformation }\end{array}$ & \multicolumn{2}{|c|}{$\begin{array}{c}d x=\mathcal{H}_{0} d y+\mathcal{N} d \tau \\
\downarrow\end{array}$} & \multicolumn{2}{|c|}{$d y=\frac{1}{\mathcal{H}_{0}} d x-\frac{\mathcal{N}}{\mathcal{H}_{0}} d t$} \\
\hline $\begin{array}{l}\text { averaged } \mathrm{CH} \\
\text { Poisson tensor }\end{array}$ & $\tilde{\mathcal{A}}_{4}$ & $\tilde{\mathcal{A}}_{3}$ & $\tilde{\mathcal{A}}_{2}$ & $\tilde{\mathcal{A}}_{1}$ \\
\hline $\mathrm{CH}$ metric & $\frac{1}{8 \mathcal{H}_{0}^{2}} g_{i i}^{K d V}(\boldsymbol{\beta})$ & $\frac{g_{i i}^{K d V}(\boldsymbol{\beta})}{4 \mathcal{H}_{0}^{2} \beta^{i}}$ & $\frac{g_{i i}^{K N V}(\boldsymbol{\beta})}{2 \mathcal{H}_{0}^{2}\left(\beta^{i}\right)^{2}}$ & $\frac{g_{i i}^{K d V}(\boldsymbol{\beta})}{\mathcal{H}_{0}^{2}\left(\beta^{i}\right)^{3}}$ \\
\hline Curvature tensor $R_{i j}^{i j}$ & $-2 \nu-\tilde{C}^{i}-\tilde{C}^{j}$ & -1 & 0 & 0 \\
\hline $\begin{array}{l}\text { averagedCH } \\
\text { Hamiltonian }\end{array}$ & $1-\frac{\nu}{\mathcal{H}_{0}}$ & $\frac{\mathcal{H}_{-1}}{\mathcal{H}_{0}}-\nu$ & $\frac{\mathcal{H}_{-2}}{\mathcal{H}_{0}}-\nu \frac{\mathcal{H}_{-1}}{\mathcal{H}_{0}}$ & $\frac{\mathcal{H}_{-3}}{\mathcal{H}_{0}}-\nu \frac{\mathcal{H}_{-2}}{\mathcal{H}_{0}}$ \\
\hline
\end{tabular}

Table 1: In the above table $\mathcal{P}_{1}, \mathcal{P}_{2}$ and $\mathcal{R}$ have been defined in remark 4.4. The averaged $\mathrm{KdV}$ Hamiltonian operators $J_{i}, i=1, \ldots, 4$ and corresponding metrics have been defined at the begining of section 4.3. The quantities $\mathcal{H}_{-s}$ are defined in (58) and (62). The averaged $\mathrm{KdV}$ Hamiltonian densities for the operators $J_{i}$, $i=1,2,3,4$ are defined modulo Casimirs. The CH Hamiltonian operators $\tilde{A}_{s}$ are defined below formula (72) and the corresponding Hamiltonians are defined in (74). The speeds $w_{+}^{i}$ are defined in (157) and the speeds $\tilde{C}^{i}$ are defined in (65). 
[3] Adler, M.; van Moerbeke, P. Completely integrable systems, Euclidean Lie algebras and curves. Adv. in Math. 38 (1980), 318-379; Adler, M.; van Moerbeke P. Linearization of Hamiltonian systems, Jacobi varieties and representation theory. Adv. in Math. 38 (1980), 318-379.

[4] Alber, M; Fedorov, Yu.N. Wave solutions of evolution equations and Hamiltonian flow on nonlinear subvarieties of generalized Jacobians J. Phys. A 33 (2000), 8409-8425.

[5] Alber, M; Camassa, R.; Fedorov, Y.; Holm, D.D.; Marsden, J.E. The complex geometry of weak piecewise smooth solutions of integrable nonlinear PDE's of shallow water and Dym type. Comm. Math. Phys. 221 (2001), no. 1, 197-227.

[6] Beals, R.; Sattinger, D. H.; Szmigielski, J. Multipeakons and the classical moment problem. Adv. Math. 154 (2000), no. 2, 229-257.

[7] Camassa R.; Holm D.D. An integrable shallow water equation with peaked solitons. Phys. Rev. Lett., 71, (1993), 1661-1664.

[8] Constantin, A.; Quasi-periodicity with respect to time of spatially periodic finite-gap solutions of the Camassa-Holm equation. Bull. Sci. Math. 122 (1998), no. $7,487-494$.

[9] Constantin, A. On the scattering problem for the Camassa-Holm equation. $R$. Soc. Lond. Proc. Ser. A Math. Phys. Eng. Sci. 457 (2001), no. 2008, 953-970.

[10] Constantin, A.; Mc Kean, H.P. A shallow water equation on the circle. Comm. Pure Appl. Math. 52 (1999), 949-982.

[11] Dobrokhotov, S.Yu.; Maslov, V.P. Multiphase asymptotics of non-linear partial differential equations with a small parameter Soviet Sci. Rev.: Math. Phys. Rev. 3 (1982), 221-311.

[12] Dubrovin, B.A.; Novikov, S.P. Hydrodynamics of weakly deformed soliton lattices. Differential geometry and Hamiltonian theory. Russian Math. Surveys 44 (1989), 35-124.

[13] Dubrovin, B.A.; Differential geometry of moduli spaces and its applications to soliton equations and to topological conformal field theory. Surveys in differential geometry: integral systems [integrable systems], 213-238, Surv. Differ. Geom., IV, Int. Press, Boston, MA, 1998. 
[14] Dubrovin, B. Geometry of 2D topological field theories. Integrable systems and quantum groups (Montecatini Terme, 1993), 120-348, Lecture Notes in Math., 1620, Springer, Berlin, 1996.

[15] Dullin, H.R.; Gottwald, G.A.; Holm, D.D. Camassa-Holm, Korteweg-de Vries5 and other asymptotically equivalent equations for shallow water waves. In memoriam Prof. Philip Gerald Drazin 1934-2002. Fluid Dynam. Res. 33, no. $1-2,73-95$.

[16] Mokhov, O.I.; Ferapontov, E.V. Nonlocal Hamiltonian operators of hydrodynamic type that are connected with metrics of constant curvature. Russian Math. Surveys 45 (1990), no. 3, 218-219.

[17] Ferapontov, E.V. Nonlocal Hamiltonian operators of hydrodynamic type: differential geometry and applications. Amer. Math. Soc. Transl. Ser.2 170, Amer. Math. Soc., Providence RI, (1995), 33-58.

[18] Ferapontov, E.V., Pavlov, M.V. Reciprocal tranformations of Hamiltonian operators of hydrodynamic type: nonlocal Hamiltonian formalism for nonlinearly degenerate systems. J. Math. Phys. 44 (2003), 1150-1172.

[19] Flaschka, H; Forest, M.G.; McLaughlin, D.W. Multiphase averaging and the inverse spectral solution of the Korteweg-de Vries equations Comm. Pure Appl. Math 33 (1980) 739-784.

[20] Fokas, A.S.; Fuchssteiner, B. Bäcklund transformations for hereditary symmetries. Nonlinear Anal. 5 (1981), 423-432.

[21] Fuchssteiner, B. Some tricks from the symmetry-toolbox for nonlinearequations: generalizations of the Camassa-Holm equation Physica D 95 (1996), 229-243.

[22] Gesztesy, F.; Helge Holden, H. Real-Valued Algebro-Geometric Solutions of the Camassa-Holm hierarchy 24 pages, Preprint http://xxx.lanl.gov/nlin.SI/0208021.

[23] Korotkin, D. Solution of matrix Riemann-Hilbert problems with quasipermutation monodromy matrices. Math.Ann. 329 (2004), no. 2, 335-364.

[24] Krichever, I.M. The averaging method for two-dimensional integrable equations. Funct. Anal. Appl. 22 (1988), no. 3, 200-213. 
[25] W.D. Hayes Group velocity and nonlinear dispersive wave propagation, Proc. Royal Soc. London Ser A. 332 (1973), 199-221.

[26] Lax, P.D.; Levermore, C.D. The small dispersion limit of the Korteweg-de Vries equation. III. Comm. Pure Appl. Math. 36 (1983), no. 6, 809-829.

[27] McKean, H.P. The Liouville correspondence between the Korteweg-de Vries and the Camassa-Holm hierarchies. Dedicated to the memory of Jrgen K. Moser. Comm. Pure Appl. Math. 56 (2003), no. 7, 998-1015.

[28] Maltsev, A.; Novikov, S.P. On the local systems Hamiltonian in the weakly non-local Poisson brackets. Phys. D 156 (2001), 53-80.

[29] Maltsev, A.Ya. Weakly-nonlocal Symplectic Structures, Whitham method, and weakly-nonlocal Symplectic Structures of Hydrodynamic Type, 64 pages, preprint http://xxx.lanl.gov/nlin.SI/0405060.

[30] Maltsev, A. Ya., private communication.

[31] Maltsev, A. Ya.; Pavlov, M. V. On Whitham's averaging method. Funct. Anal. Appl. 29 (1995), no. 1, 6-19.

[32] Pavlov, M. V.; Tsarev, S. P. Tri-Hamiltonian structures of Egorov systems of hydrodynamic type. (Russian) Funktsional. Anal. i Prilozhen. 37 (2003), no. 1, 38-54, 95; translation in Funct. Anal. Appl. 37 (2003), no. 1, 32-45.

[33] Tian, F.R. Oscillations of the zero dispersion limit of the Korteweg-de Vries equation. Comm. Pure Appl. Math. 46 (1993), 1093-1129.

[34] Tian, F.R. The initial value problem for the Whitham averaged system. Comm. Math. Phys. 166 (1994), 79-115.

[35] Tsarev, S.P. Poisson brackets and one-dimensional Hamiltonian systems of hydrodynamic type. Dokl. Akad. Nauk. SSSR 282 (1985), 534-537.

[36] Vanhaecke P. Integrable systems and symmetric product of curves. Math. Z., 227 (1998), no. 1, 93-127.

[37] Whitham, G.B. A general approach to linear and non-lin ear dispersive waves using a Lagrangian J. Fluid. Mech. 22 (1965), 273-283. 